\title{
ANAIS DO XIII CONGRESSO MÉDICO ACADÊMICO
}

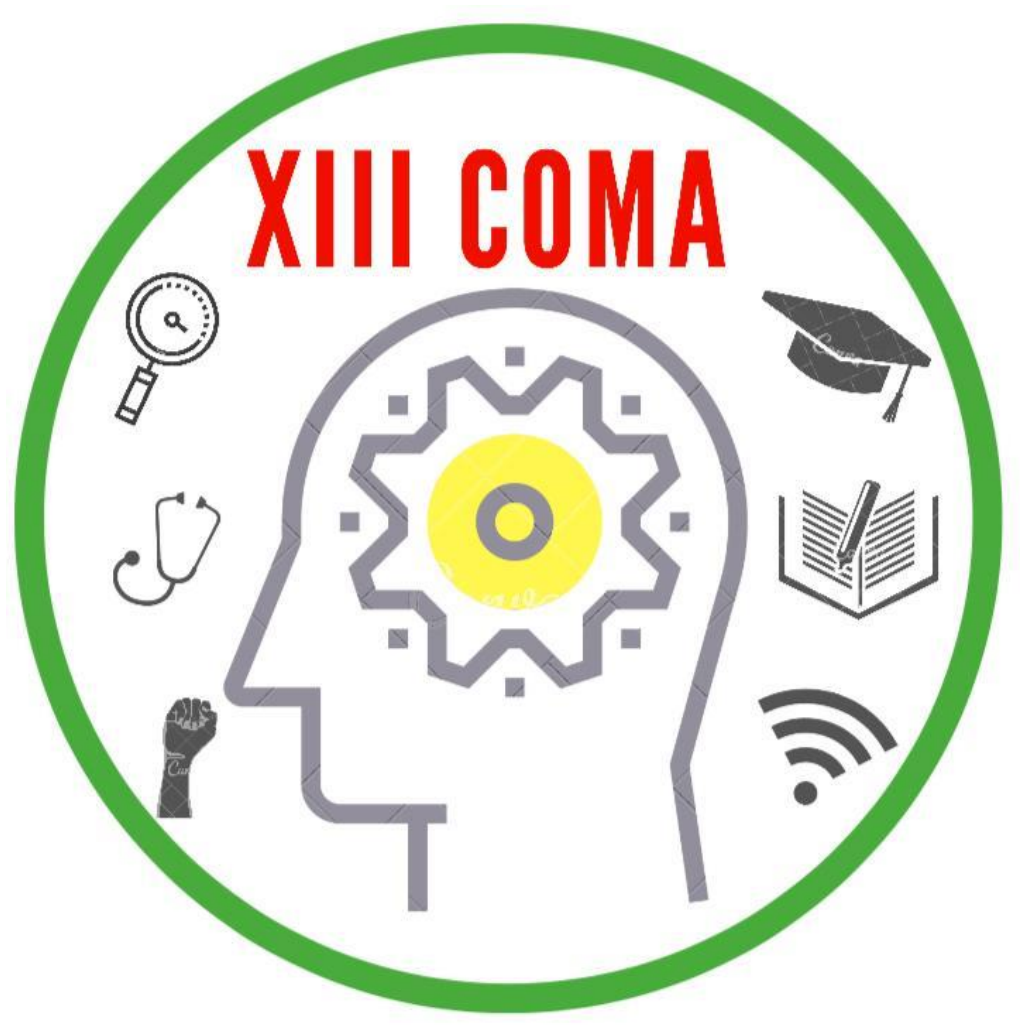

APOIO:

ACERVO www.acervomais.com.br

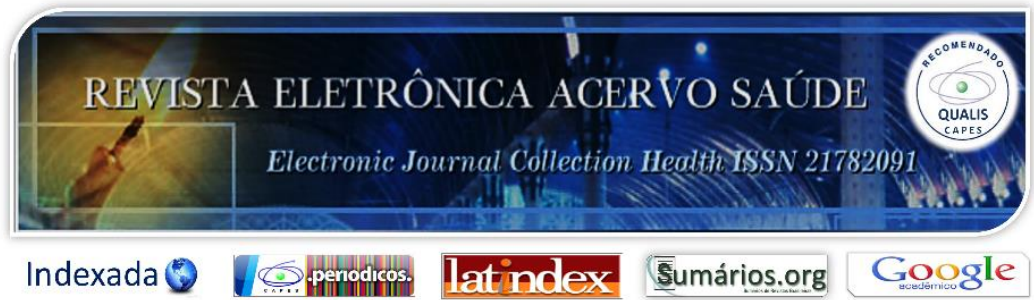

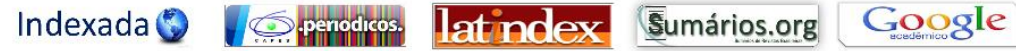




\section{ORGANIZADORES DO XII CONGRESSO MÉDICO ACADÊMICO}

- Ana Carolina Delgado Malvaccini Mendes;

- André Coutinho Alves Mingote;

- Antonio José de Oliveira Machado;

- Augusto José de Oliveira Santos;

- Daniel Nolasco Gouveia;

- Gabriel Vinícius Trindade de Abreu;

- Gabriel Zeferino de Oliveira Souza;

- Isabella Oliveira Lanzieri;

- Jussara Ramos Ribeiro;

- Letícia Sabrina Neves;

- Luíza Campos de Paula;

- Maria Emília Batista Rosa;

- Petrus Ferreira Renó;

- Tainá Diana Rodrigues;

- Tayná Magalhães de Almeida. 


\section{INTEGRANTES DA COORDENAÇÃO CIENTÍFICA \\ Daniel Nolasco Gouveia \\ Coordenador do XIII Congresso Médico Acadêmico}

- Ana Carolina Delgado Malvaccini Mendes;

- Antonio José de Oliveira Machado;

- Augusto José de Oliveira Santos;

- Gabriel Vinícius Trindade de Abreu;

- Gabriel Zeferino de Oliveira Souza;

- Isabella Oliveira Lanzieri;

- Luíza Campos de Paula;

- Petrus Ferreira Renó;

- Tainá Diana Rodrigues;

- Tayná Magalhães de Almeida.

\section{ORGANIZADOR DOS ANAIS}

- Petrus Ferreira Renó.

\section{INTEGRANTES DA BANCA AVALIADORA}

- Lorena Nagme de Oliveira Pinto;

- Mário Círio Nogueira;

- Maximiliano Ribeiro Guerra. 


\title{
PROGRAMAÇÃO DO XIII CONGRESSO MÉDICO ACADÊMICO
}

\author{
GRANDES ÁREAS:
}

EDUCAÇÃO

PESQUISA

EXTENSÃO

\begin{tabular}{|c|l|l|}
\hline \multicolumn{2}{|c|}{ DIA 1-03.04.2019 } \\
\hline HORÁRIO & \multicolumn{1}{|c|}{ PALESTRA } & \multicolumn{1}{|c|}{ PALESTRANTE } \\
\hline 19:00-19:40 & Metacognição: estudando menos e aprendendo mais & Rodolfo Dias Correa \\
\hline 19:50-20:30 & Feedback: como aprender com os erros e os acertos & Oscarina da Silva Ezequiel \\
\hline 20:40-21:20 & $\begin{array}{l}\text { Metodologias Ativas: como o aluno pode otimizar o seu } \\
\text { aprendizado }\end{array}$ & Alice Belleigoli Rezende \\
\hline 21:30-22:30 & Coffee Break & \multicolumn{2}{|l}{} \\
\hline
\end{tabular}

\begin{tabular}{|c|l|l|}
\hline \multicolumn{2}{|c|}{ DIA 2-04.04.2019 } \\
\hline HORÁRIO & \multicolumn{1}{|c|}{ PALESTRA } & \multicolumn{1}{|c|}{ PALESTRANTE } \\
\hline 19:00-19:40 & O papel da pesquisa na graduação & Giancarlo Lucchetti \\
\hline 19:50-20:30 & Revisão de literatura: princípios básicos e como fazer & Renato Erothildes Ferreira \\
\hline 20:40-21:20 & Experimentação animal: quando fazer e princípios básicos & $\begin{array}{l}\text { Bárbara Bruna Abreu de } \\
\text { Castro }\end{array}$ \\
\hline 21:30-22:30 & Coffee Break & \\
\hline
\end{tabular}

\begin{tabular}{|l|l|}
\hline \multicolumn{2}{|l|}{ DIA 3 - 05.04.2019 } \\
\hline HORÁRIO & ATIVIDADE \\
\hline 13:00-15:00 & Apresentação de pôsteres - Etapa 1 \\
\hline 15:00-17:00 & Apresentação de pôsteres - Etapa 2 \\
\hline
\end{tabular}

\begin{tabular}{|c|c|c|}
\hline \multicolumn{3}{|c|}{ DIA 3-05.04.2019 } \\
\hline HORÁRIO & PALESTRA & PALESTRANTE \\
\hline 19:00-19:20 & Marketing médico & $\begin{array}{l}\text { Medic Jr.: Daniel Coelho } \\
\text { Pereira e Pedro Freitas Melo }\end{array}$ \\
\hline $19: 30-19: 50$ & Prática médica internacional: como e porque fazer & $\begin{array}{l}\text { CLEV-UFJF: Petrus Ferreira } \\
\text { Renó }\end{array}$ \\
\hline $20: 00-20: 20$ & Pesquisa médica internacional: como e porque fazer & $\begin{array}{l}\text { IFMSA-Brazil: Lucas Mizrahy } \\
\text { Lima }\end{array}$ \\
\hline $20: 30-20: 50$ & Oportunidades de Internacionalização dentro da UFJF & $\begin{array}{l}\text { Diretoria de Relações } \\
\text { Internacionais: Bárbara Inês } \\
\text { Ribeiro Simões Daibert }\end{array}$ \\
\hline $21: 00-21: 30$ & $\begin{array}{l}\text { Mesa redonda: desafios da internacionalização no curso de } \\
\text { medicina }\end{array}$ & $\begin{array}{l}\text { Bárbara Inês Ribeiro Simões } \\
\text { Daibert, Lucas Mizrahy Lima, } \\
\text { Petrus Ferreira Renó, e } \\
\text { Rafael Kenji Fonseca Hamada }\end{array}$ \\
\hline $21: 40-22: 40$ & mee or & \\
\hline
\end{tabular}

\begin{tabular}{|l|c|}
\hline \multicolumn{2}{|c|}{ DIA 4-06.04.2019 } \\
\hline HORÁRIO & ATIVIDADE \\
\hline
\end{tabular}


07:00-09:05 Workshop das ligas - Etapa 1

09:10-09:50 Café com prosa (DENEM)

09:55-11:35 Workshop das ligas - Etapa 2
DENEM: Nathalia Julie Soares Resende;

Pedro Rocha Badô 


\section{PATROCINADORES}

- Côrtes Villela;

- Freeway;

- Libbs;

- MedClass;

- Phormar;

- ProSport;

- UNICRED. 


\section{SUMÁRIO}

APRESENTAÇÃ 0 ........................................................................................ 8

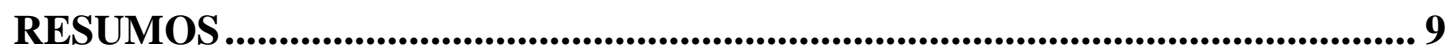

ELABORAÇÃO DE PROTOCOLOS CLÍNICOS E CIRÚRgICOS POR ACADÊMICOS NO HPS: UM RELATO DE EXPERIÊNCIA ............................. 10

RELATO DE EXPERIÊNCIA: I SIMPÓSIO DE SINTOMATOLOGIA CLÍNICA DA IFMSA BRAZIL UFJF 12

TRABALHANDO HABILIDADES NARRATIVAS NO CONTEXTO DA EDUCAÇÃO MÉDICA- UM RELATO DE EXPERIÊNCIA................................ 14

EMERGÊNCIAS MÉDICAS EM ODONTOLOGIA: A REALIDADE DAS GRADUAÇÕES EM MINAS GERAIS 16

O DEBATE INCIPIENTE DA VIOLENCIA NO MEIO ACADÊMICO E SUA SUPERAÇÃO ATRAVÉS DA LAMFAC-UFJF. 18

PERFIL EPIDEMIOLÓGICO DOS PACIENTES COM NÓDULOS TIREOIDIANOS DO AMBULATÓRIO DE ENDOCRINOLOGIA-TIREOIDE DA UFJF 20

APRENDIZADO MELHORADO POR PROVAS COMO METODOLOGIA DE ESTUDO: UMA REVISÃO NARRATIVA 22

RASTREAMENTO ORGANIZADO DOS CÂNCERES DE MAMA E COLO DO ÚTERO EM JUIZ DE FORA-MG: IMPLANTAÇÃO DE UM PROJETO PILOTO UM RELATO DE EXPERIÊNCIA 26

RELATO DE EXPERIÊNCIA: COMUNIDADE SAUDÁVEL - PROMOVENDO SAÚDE E REDUZINDO VULNERABILIDADES ATRAVÉS DO ACESSO E INTERSETORIALIDADE. 28

PERDENDO O MEDO DO MÉDICO COM O PROJETO DE EXTENSAO HOSPITAL DE URSINHOS: RELATO DE EXPERIÊNCIA 30

RELATO DE EXPERIÊNCIA: IMPORTÂNCIA DO CONTATO PRECOCE DO ESTUDANTE DE MEDICINA COM O AMBIENTE MÉDICO . 32

ABORDAGEM TERAPÊUTICA DA CRIANÇA COM TRANSTORNO DO ESPECTRO AUTISTA: ATENDIMENTO AMPLIADO AOS PAIS................... 34

PREVENÇÃO PARASITOSES INTENTINAIS E PEDICULOSE EM ESCOLARES: UM RELATO DE EXPERIÊNCIA 36

RELATO DE CASO: SÍFILIS CONGÊNITA E SEPSE NEONATAL EM RECÉMNASCIDO A TERMO 38

REVISÃO DE LITERATURA: IMPACTO DA AVALIAÇÃO DA ARTÉRIA CARÓTIDA NA ESTRATIFICAÇÃO DO RISCO CARDIOVASCULAR EM HIPERTENSOS. 40 
EDUCAÇÃO CONTRA O TABACO(EAT) NA PREVENÇÃO DO TABAGISMO: UM RELATO DE EXPERIÊNCIA 42

CONHECIMENTOS DE USUÁRIAS DA ATENÇÃO BÁSICA DE JUIZ DE FORA SOBRE O RASTREAMENTO DAS NEOPLASIAS FEMININAS 44

RELATO DE CASO: DOENÇA CELÍACA EM ADULTOS. 46

AMBULATÓRIO DE ATENÇÃO A CRIANÇAS E ADOLESCENTES COM TRANSTORNO DO ESPECTRO AUTISTA: RELATO DE EXPERIÊNCIA. 48 PROJETO DE EXTENSÃO "SAÚDE E ESPIRITUALIDADE" ........................50 RELATO DE CASO: LINFOMA NÃO HODGKIN DE CÉLULAS DO MANTO 52

RELATO DE CASO: SÍNDROME DE TAKOTSUBO, A CARDIOMIOPATIA EMERGENTE DA ERA MODERNA 54

RELATO DE CASO: LESÃO MULTILIGAMENTAR DO JOELHO COM TRATAMENTO POR ENXERTO AUTÓLOGO COM RETORNO AO ESPORTE 56

RELATO DE EXPERIÊNCIA: CONSCIENTIZANDO A POPULAÇÃO SOBRE FIBROSE CÍSTICA 58

RELATO DE EXPERIÊNCIA: APRENDIZADO PRÁTICO DE DISCENTES DE MEDICINA EM CENTRO DE REFERÊNCIA EM FIBROSE CÍSTICA ........59 MELHORES TRABALHOS 61 


\section{APRESENTAÇÃO}

O Congresso Médico Acadêmico é realizado anualmente pelo Diretório Acadêmico Silva Mello da Faculdade de Medicina da Universidade Federal de Juiz de Fora (UFJF). Hoje, encontra-se em sua décima terceira edição. Aborda assuntos que são de interesse acadêmico e médico, além de tratar sobre questões sociais, como saúde da população marginalizada, racismo, fraude de cotas em universidades públicas e saúde mental dos estudantes. O evento ficou caracterizado pela excelência acadêmica, graças ao alto nível dos conferencistas e ao apoio da Faculdade de Medicina da UFJF.

Nesse ano, o tema principal do congresso foi "a busca pelo conhecimento". Os palestrantes abordaram temas acerca do tripé da graduação: ensino, pesquisa e extensão. Os objetivos das palestras foram mostrar como nós, estudantes, podemos aproveitar os pontos levantados nas mesmas em nosso aprendizado e em nossa formação. 


\title{
ELABORAÇÃO DE PROTOCOLOS CLÍNICOS E CIRÚRGICOS POR ACADÊMICOS NO HPS: UM RELATO DE EXPERIÊNCIA
}

\author{
Oliveira Guimarães ${ }^{2}$; Sérgio Castro Pontes ${ }^{3}$. \\ ${ }^{1}$ Graduação; Faculdade de Medicina da Universidade Federal de Juiz de Fora; \\ ${ }^{2}$ Graduação; Faculdade de Medicina da Universidade Federal de Juiz de Fora; \\ ${ }^{3}$ Docente da Faculdade de Medicina da Universidade Federal de Juiz de Fora.
}

Éuqor Antônio Pereira Vianella ${ }^{1}$; Isabele Nogueira Silva²; Janaina Matias Moreira²; Lívia de

Autor para correspondência:

Éuqor Antônio Pereira Vianella

Email: euqorjf@gmail.com

\section{RESUMO}

Introdução: A Urgência e Emergência são caracterizadas pelo atendimento imediato, ampla capacitação técnica, habilidade profissional e uso de recursos tecnológicos visando oferecer atendimentos resolutivos e humanizados. Para maximizar a efetividade nesse contexto, foram criados métodos de padronização de condutas por meio de protocolos clínicos e cirúrgicos. Os dados sobre consensos utilizados são majoritariamente importados de países do Hemisfério Norte, visto que no Brasil esses são escassos. Nos EUA, uma revisão sistemática sobre a efetividade dos protocolos mostrou que 93\% das 59 avaliações publicadas de protocolos clínicos revelaram melhorias significativas no sistema de saúde ${ }^{1}$. Objetivos: Este trabalho tem como objetivo relatar a experiência da elaboração de protocolos clínicos e cirúrgicos no Hospital Doutor Mozart Teixeira, em Juiz de Fora - MG, almejando aprimorar o aprendizado prático dos estudantes de medicina, redução de custos hospitalares, otimizar condutas e assistência médica. Relato de Experiência: O estágio no HPS proporcionou a participação de um projeto de elaboração de protocolos, dentre eles o de Pneumonia Adquirida na Comunidade - PAC, que de acordo com os registros de internação do Departamento de Informática do SUS, foi a segunda causa de hospitalizações no Sudeste em 2007². Em 2016 esteve na $4^{\circ}$ posição no ranking de causas de morte no Brasil ${ }^{3}$. A base de dados utilizada foi cedida pelo professor orientador e pautada em revisões de literatura sobre o assunto e as suas formas de investigação diagnóstica e tratamento. Após a leitura e vivência no estágio na instituição, foram produzidos fluxogramas que guiassem o diagnóstico clínico e a conduta conforme avaliação da gravidade do doente. O protocolo foi revisado para promover acesso rápido a conduta de acordo com os recursos disponíveis no HPS. Durante esse período foram agregados vários atributos ao chamado currículo oculto, o qual cresce a cada nova experiência na prática médica. Discussão: A elaboração dos protocolos pelos acadêmicos mostrou-se uma atividade de baixo custo, tempo reduzido além de contar com bibliografia atualizada sobre o assunto. Procurou-se conciliar propedêutica e tratamento conforme as ferramentas disponíveis no HPS. No contexto de um serviço público, entretanto, alguns entraves surgem durante a tentativa de implementação de um protocolo. $\mathrm{O}$ mau gerenciamento dos insumos, falta de fiscalização, assim como de promoção e incentivo a educação continuada, atos médicos negligentes aliados a ausência de engajamento da equipe a propostas mais sustentáveis para o sistema são protagonistas do insucesso do sistema. Resultados: Ao término das atividades, obtiveram-se protocolos clínicos e cirúrgicos sobre temáticas mais prevalentes nos atendimentos realizados pelos acadêmicos durante estágio. Confeccionou-se um protocolo de atendimento a PAC com estrutura sucinta e linguagem prática, pautada na realidade do hospital. Desse modo, esperou-se aperfeiçoar a assistência médica prestada na instituição, com foco na factibilidade das condutas e padronização dos tratamentos da população. Conclusão: Por fim, a realização dos protocolos pelos acadêmicos propôs otimizar os serviços prestados pela instituição, garantindo qualidade e equidade nos 


\section{ANAIS DO XIII CONGRESSO MÉDICO ACADÊMICO, 2019; 08-61}

atendimentos. Ademais, tal experiência mostrou-se agregadora na formação científica, profissional e pessoal dos acadêmicos de medicina.

Palavras-chave: Protocolos. Acadêmicos. Urgência.

\section{Referências:}

1. Grimshaw, J. M.; Russel, I. T. Effect of clinical guidelines on medical practice: a systematic review of rigorous evaluations. Lancet, v.342, n. 8883, nov 27, p. 1317 -1322, 1993.

2. Donalisio, M. R., Arca, C. H. M., \& Madureira, P. R. D. (2011). Perfil clínico, epidemiológico e etiológico de pacientes com pneumonia adquirida na comunidade internados em um hospital geral da microrregião de Sumaré, SP. J Bras Pneumol, 37(2), 200-8.

3. Ministério da Saúde. Sistema de informações sobre mortalidade. Disponível em: < http://tabnet.datasus.gov.br/cgi/tabcgi.exe?sim/cnv/obt10uf.def> Acesso em: 24 mar.2019. 


\section{RELATO DE EXPERIÊNCIA: I SIMPÓSIO DE SINTOMATOLOGIA CLÍNICA DA IFMSA BRAZIL UFJF}

Ítalo Pereira ${ }^{1}$; Maria Coimbra Pereira de Jorge ${ }^{2}$; Maria Paula Coelho de Sousa Lima ${ }^{2}$; Gabriella Altomare Andrade²; José Luiz de Jorge Júnior ${ }^{3}$

${ }^{1}$ Graduação; Universidade Federal de Juiz de Fora;

${ }^{2}$ Graduação; Universidade Federal de Juiz de Fora;

${ }^{3}$ Médico atuante no Hospital Albert Sabin.

Autor para correspondência: Ítalo Pereira

Email: italo_pereira@ outlook.com

\section{RESUMO}

INTRODUÇÃO: A Faculdade de Medicina da Universidade Federal de Juiz de Fora (UFJF) adota uma metodologia de ensino tradicional, dividida por matérias referentes às grandes especialidades e com temática baseada em patologias 1 . Com aulas ministradas com especialistas, muitas vezes o foco foge do geral e se volta para o específico. Concomitantemente, estágios em Unidades Básicas de Saúde (UBS) são realizados e os alunos se deparam com um paciente sem um diagnóstico, mas que trazem queixas das mais diversas $^{2,3,4,5,6}$. Nesse contexto, a IFMSA Brazil UFJF propôs a realização do I Simpósio de Sintomatologia Clínica da IFMSA Brazil UFJF, o qual visou a sistematização de ao menos alguns dos principais, conteúdos em saúde. OBJETIVOS: Promover o raciocínio baseado em diagnósticos sindrômicos e estimular os estudantes a fazerem estudos sistematizados, além de esclarecer os principais diagnósticos diferenciais das queixas mais comuns em UBSs e UPAs. RELATO DE EXPERIÊNCIA: O I Simpósio de Sintomatologia Clínica da IFMSA Brazil UFJF foi um evento que contou com a participação de mais de 180 inscritos e de oito professores. O simpósio abrangeu oito palestras sobre diferentes especialidades médicas, cuja pergunta central foi "Por que o (a) senhor (a) está me procurando hoje?". As palestras se basearam em queixas principais que leva os pacientes a buscarem auxílio médico nas principais áreas da profissão. O evento ocorreu de $18 \mathrm{~h} 00$ às $22 \mathrm{~h} 00$ nos dias 28 e 29 de agosto de 2018. Cada palestra teve duração de 50 minutos. Os temas abordados foram: Método Clínico; Icterícia; Cefaleia; Neurociências e Psicanálise; Função Renal; Dispneia; Dor em Membros e Vacinas. Como avaliação de impacto utilizamos um questionário no Google Forms que foi encaminhado aos participantes da ação no último dia do evento, sendo conferidas notas de 1 a 5. RESULTADOS E DISCUSSÃO: Foi observada uma ampliação no conhecimento sobre algumas das principais queixas existentes na atenção básica e unidades de pronto atendimento, complementando a formação médica da comunidade acadêmica local. Os estudantes das etapas iniciais do curso de Medicina puderam ter um resumido apanhado sobre estas queixas, aprendendo a lidar melhor com os sinais e sintomas dos pacientes que podem chegar até os mesmos durante a graduação e durante o exercício da profissão, introduzindo-os brevemente no ciclo clínico do curso. Do mesmo modo, estudantes das etapas intermediárias e finais do curso puderam revisar o que já aprenderam, focando-se nos pontos mais importantes a se lembrar quando se trata dos temas abordados. O evento foi inovador à medida que se mudou o foco da abordagem médica de patologias para queixas, $\mathrm{o}$ que chamou a atenção dos palestrantes e dos alunos presentes. A principal sugestão ou crítica quanto ao simpósio foi a inserção de mais temas nas palestras e a realização do evento mais vezes nos próximos semestres. CONCLUSÃO: A repercussão do I Simpósio de Sintomatologia Clínica da IFMSA Brazil UFJF foi maior do que o esperado, contando com a presença de alunos de três faculdades de medicina de Juiz de Fora e expandindo a discussão 


\section{ANAIS DO XIII CONGRESSO MÉDICO ACADÊMICO, 2019; 08-61}

sobre uma educação médica de qualidade. O evento teve boa avaliação e alcançou seu objetivo ao possibilitar o contato dos estudantes com algumas das principais queixas clínicas presentes nas UAPSs e UPAs. Além disso, o evento foi uma ótima forma de divulgar a IFMSA Brazil UFJF.

Palavras-chave: educação médica; sintomas; clínica médica.

\section{Referências:}

1. MOUTINHO, I. L. D. et al. Projeto pedagógico de curso - Faculdade de Medicina da Universidade Federal de Juiz De Fora. 2015. Disponível em: <http://www.ufjf.br/medicina/files/2015/04/PPC_FAMED2015_.pdf>. Acessado em: 23/03/2019.

2. BRASIL. Ministério da Saúde. Secretaria de Atenção à Saúde. Departamento de Atenção Básica. Acolhimento à demanda espontânea: queixas mais comuns na Atenção Básica. Cadernos de Atenção Básica. Ministério da Saúde, Secretaria de Atenção à Saúde, Departamento de Atenção Básica. - 1. ed.; 1. Reimp. - Brasília: Ministério da Saúde, 2013

3. DINIZ, A. S. et al. Demanda clínica de uma unidade de pronto atendimento, segundo o protocolo de Manchester. Revista Eletrônica de Enfermagem, v. 16, n. 2, p. 312-320, 2014.

4. OLIVEIRA, G. N. et al. Perfil da população atendida em uma unidade de emergência referenciada. Revista Latino-Americana de Enfermagem, v. 19, n. 3, p. 1-9, 2011.

5. PIMENTEL, Í. R. S. et al. Caracterização da demanda em uma Unidade de Saúde da Família. Revista Brasileira de Medicina de Família e Comunidade, v. 6, n. 20, p. 175-181, 2012. 


\title{
TRABALHANDO HABILIDADES NARRATIVAS NO CONTEXTO DA EDUCAÇÃO MÉDICA- UM RELATO DE EXPERIÊNCIA
}

\author{
Ramos ${ }^{4}$. \\ ${ }^{1}$ Graduação; Faculdade de Medicina da Universidade Federal de Juiz de Fora; \\ ${ }^{2}$ Graduação; Faculdade de Medicina da Universidade Federal de Juiz de Fora; \\ ${ }^{3}$ Graduação; Faculdade de Letras da Universidade Federal de Juiz de Fora; \\ ${ }^{4}$ Docente da Faculdade de Medicina da Universidade Federal de Juiz de Fora.
}

Ana Flávia de Seixas Salomão ${ }^{1}$; Francieli do Carmo Pereira Oliveira ${ }^{2}$; Milena Passini

Medeiros $^{2}$; Luiza Ferreira Defavere ${ }^{2}$; Diogo Alvim Barbara ${ }^{3}$; Andreia Aparecida de Miranda

Autor para correspondência:

Ana Flávia de Seixas Salomão

Email: anaflaviaseixas@gmail.com;

\section{RESUMO}

Introdução: A assistência à saúde encontra-se repleta de contradições e desafios. Se, por um lado, as novas tecnologias permitem recursos diagnósticos e terapêuticos mais acurados, por outro, profissionais de saúde carecem da capacidade de oferecer conforto e amparo àqueles que sofrem. ${ }^{1}$ Nesse sentido, as habilidades cognitivas e científicas precisam ser complementadas pelo que se denominam habilidades narrativas, que incorporam a capacidade de ouvir histórias, absorver e interpretar seu significado mais amplo, assim como comover-se com a dor do outro. ${ }^{2}$ Objetivos: $O$ presente trabalho objetiva descrever a experiência do projeto de extensão "Contando Histórias" que buscou oferecer aos acadêmicos de Medicina uma introdução e treinamento destas habilidades narrativas e, ao mesmo tempo, uma abordagem em saúde mais humanizada para os usuários do Hospital Universitário da Universidade Federal de Juiz de Fora (UFJF). Relato de experiência: A metodologia do projeto incluiu a realização de entrevistas, no período de outubro de 2018 a março de 2019, com os pacientes do Hospital Universitário da UFJF. Essas abordagens eram realizadas pelos acadêmicos participantes, incorporando conhecimentos da área de Linguística e Literatura aos conceitos da Semiologia Médica, para enriquecer o entendimento e interpretação das narrativas orais fornecidas pelos usuários. Buscou-se abordar, além da história clínica tradicional, o trajeto do paciente no sistema de saúde, suas impressões e sentimentos sobre o adoecimento e os mecanismos de coping utilizados. Concomitantemente, foram realizadas reuniões de estudo e aprimoramento sobre o tema Narrativas em Saúde, com participantes dos cursos de Medicina e Letras. Resultados e discussão: Como resultado, foram entrevistados 40 pacientes, buscando-se utilizar habilidades narrativas, em conjunto com as habilidades cognitivas e científicas. Os acadêmicos puderam aprender sobre a importância de respeitar e valorizar a fala livre do paciente, englobando a linguagem verbal e não verbal, para que se possa maximizar a compreensão de todas as informações que ela traz: as características singulares daquele paciente, os pontos que mais trazem sofrimento, as estratégias de enfrentamento da doença que devem ser encorajadas e os maiores empecilhos sociais, ambientais e culturais à realização adequada de um tratamento. Conclusão: Nesse sentido, pode-se concluir que o Projeto "Contado Histórias" representou uma oportunidade de treinamento dos acadêmicos em habilidades pouco abordadas na graduação em Medicina, que contribuíram para a humanização do cuidado e a melhoria da relação médico-paciente.

Palavras-chave: Educação Médica; Medicina Narrativa; Relações Comunidade-Instituição. 


\section{ANAIS DO XIII CONGRESSO MÉDICO ACADÊMICO, 2019; 08-61}

\section{Referências:}

1. CHARON, Rita. Narrative Medicine: A Model for Empathy, Reflection, Profession, and Trust. JAMA.2001;286(15):1897-1902. doi:10.1001/jama.286.15.1897

2. CHARON, Rita. Narrative medicine: form, function, and ethics. Annals of Internal Medicine, 2001, 134.1: 83-87. 


\title{
EMERGÊNCIAS MÉDICAS EM ODONTOLOGIA: A REALIDADE DAS GRADUAÇÕES EM MINAS GERAIS
}

\author{
Luan Viana Faria ${ }^{1}$; Yuri de Lima Medeiros ${ }^{1}$; Danielle Fernandes Lopes ${ }^{1}$; Ana Luísa Soares \\ Costa $^{2}$; Eduardo Machado Vilela ${ }^{3}$. \\ ${ }^{1}$ Acadêmico de Odontologia da Universidade Federal de Juiz de Fora; \\ 2 Acadêmico de Medicina da Universidade Federal de Juiz de Fora; \\ ${ }^{3}$ Professor associado ao Departamento de Clínica Odontológica da Faculdade de Odontologia da Universidade \\ Federal de Juiz de Fora.
}

Autor para Correspondência: Luan Viana Faria

Email: luanvfaria13@hotmail.com

\begin{abstract}
RESUMO
Introdução e Objetivos: Os primeiros socorros são definidos como um atendimento temporário e imediato de uma pessoa que está ferida ou que adoece repentinamente, bem como, se insere o atendimento no domicílio quando não se pode ter acesso a uma equipe de resgate ou enquanto os técnicos em emergência médica não chegam ao local ${ }^{1,2}$. $\mathrm{O}$ cirurgiãodentista lida cotidianamente com o risco de se deparar com emergências médicas relacionadas à saúde sistêmica de seus pacientes ${ }^{3}$. Muitas são as situações que ocorrem, desde quadros menos graves, como síncopes e lipotimias, até situações de extrema gravidade, como os casos de parada cardiorrespiratória ${ }^{2,4}$. A partir disso, este trabalho tem como objetivo realizar uma pesquisa nas faculdades de Odontologia de Minas Gerais reconhecidas pelo Ministério da Educação e Cultura (MEC), e verificar se os cursos apresentam na sua grade curricular a disciplina de Primeiros socorros, e caso exista, se é obrigatória ou optativa, teórica ou prática, e a carga horaria da mesma. Material e Métodos: As grades curriculares das faculdades foram avaliadas e os dados coletados analisados descritivamente, sendo excluídas desta pesquisa as faculdades que não disponibilizam a grade curricular e as que ainda não tiveram o curso inaugurado. Resultados e Discussão: Dentre as 61 universidades cadastradas em Minas Gerais, 51 foram incluídas no estudo, sendo que 44 são universidades particulares e sete são públicas. Dentre elas, somente 10 apresentam a disciplina de Primeiros Socorros, sendo nove privadas e uma universidade federal. Após analise, verificou-se que nove apresentam a disciplina com caráter obrigatório e somente uma faculdade apresenta a disciplina com caráter optativo. A análise da carga horária reservada para esta disciplina apresentou uma média de 53 horas, sendo que somente nove faculdades disponibilizaram esta informação. Além disso, três possuem atividades práticas relacionadas ao conteúdo ministrado, três possuem a metodologia de ensino à distância conjugada com aulas teóricas, duas possuem somente aulas teóricas e duas não disponibilizaram a metodologia de ensino da matéria. Em outros estudos, percebeu-se que o conhecimento do cirurgião-dentista na temática é limitado, sendo este um tema subvalorizado na formação acadêmica e, por isso, não aprofundado ${ }^{3,4,5}$. Conclusão: Diante dos fatos, pode-se concluir que há carência na formação de alunos de graduação com um conhecimento básico sobre emergências médicas, não sendo capacitados para atuar na prevenção de possíveis danos que possam ocorrer em seu ambiente de trabalho. Assim, é importante não só a reformulação curricular, como também a discussão do assunto nas equipes odontológicas, estimulando a formação nesta área.
\end{abstract}

Palavras-chave: Primeiros socorros, Odontologia, Universidades. 


\section{Referências:}

1- ANDERS, P. et al. The nature and frequency of medical emergencies among patients in a dental school setting. Journal of a Dental Education, v. 74, n. 4, p. 392-396, 2010.

2- ARAGON, C. Understanding the patient with epilepsu and seizures in the dental practice. Journal of Canadian Dental Association, v. 73, n. 1, p. 71-76, 2007.

3- ARSATI, F. Brazilian Dentists' Attitudes About Medical Emergencies During Dental Treatment. Journal of Dental Education, v. 74, n. 6, p. 661-666, 2010.

4- JODALLI, P.; ANKOLA, A. Evaluation of knowledge, experience and perceptions about medical emergencies amongst dental graduates (Interns) of Belgaum City. Journal Clin Exp Dent, v. 4, n. 1, p. 14-18, 2012.

5- LÚCIO, P.; BARRETO, R. Emergência médica no consultório odontológico e a (in)segurança dos profissionais, Revista Brasileira de Ciência e Saúde, v. 16, n. 2, p. 267-272, 2012. 


\title{
O DEBATE INCIPIENTE DA VIOLENCIA NO MEIO ACADÊMICO E SUA SUPERAÇÃO ATRAVÉS DA LAMFAC-UFJF
}

\author{
Tiago de Jesus Oliveira ${ }^{1}$; Ana Luísa Soares Costa ${ }^{1}$; Mikaela Santos Mascarenhas ${ }^{1}$; Lorenha \\ Pinholi de Morais ${ }^{1}$; Mário Círio Nogueira ${ }^{2}$ \\ ${ }^{1}$ Graduação; Faculdade de Medicina, Universidade Federal de Juiz de Fora; \\ ${ }^{2}$ Orientador; Departamento de Saúde Coletiva, Faculdade de Medicina, Universidade Federal de Juiz de Fora.
}

Autor para correspondência:

Thiago Jesus Oliveira

E-mail: jesustiagooliveira@gmail.com

\section{RESUMO}

Introdução: A violência é uma das problemáticas mais presentes na Atenção Primária à Saúde (APS), devido a seu caráter de porta de entrada para os problemas da comunidade. Além de cuidar dos agravos físicos e emocionais decorrentes dessa temática, a APS também é responsável pela prevenção e pela promoção da saúde, em busca do conceito ampliado de bem-estar individual e coletivo ${ }^{1}$. A Liga Acadêmica de Medicina de Família e Comunidade (LAMFAC-UFJF) visa trazer para o ambiente acadêmico discussões que, geralmente são pouco abordadas na faculdade, e ao mesmo tempo convidar a população para conhecer mais sobre estes temas. Objetivos: Relatar as experiências vivenciadas pelos membros da LAMFAC-UFJF durante um evento de educação em saúde, cujo o público era composto por acadêmicos e profissionais vinculados à saúde e áreas afins, bem como mencionar as situações vivenciadas por estes nos campos de prática. Relato de experiência: Diante da elevada taxa de violência nas comunidades acompanhadas pelos membros da Liga e o debate acadêmico insuficiente acerca da identificação e conduta dessas situações, a LAMFAC-UFJF promoveu o simpósio "Abordagem da Violência na Atenção Básica". A escolha dos temas foi feita após discussões entre os 41 integrantes da Liga, que estagiam ativamente em dez Unidades Básicas de Saúde (UBS's) na cidade de Juiz de Fora. Foram realizadas cinco palestras que tratavam da identificação e conduta pelo profissional de saúde em casos de agressão contra a mulher, o idoso, o jovem negro, a população LGBT+ e a criança ${ }^{2}$. O evento transcendeu o meio acadêmico através do convite à população nas UBS's e pela parceria com a Prefeitura de Juiz de Fora. Resultados e discussão: Os participantes saíram mais informados e preparados para abordar situações que possam surgir nas UBS's, não apenas reconhecendo-as, mas também, ativando os mecanismos jurídicos e de amparo social pertinentes. Foram evidenciados os meios para que casos de violência sejam identificados, abordados e não perpetuados, de maneira complementar à formação curricular dos cursos de saúde, incipientes quanto ao tema. Conclusão: A Violência é um tema extremamente prevalente na prática clínica, porém, muitas vezes negligenciada pela formação acadêmica. Nesse sentido, o simpósio ampliou o debate sobre o tema, transcendendo a universidade e incorporando a população e os órgãos públicos nas discussões, sob a ótica de diversas áreas da saúde, privilegiando a interdisciplinaridade.

Palavras-chave: Saúde da família; Prevenção à violência; Educação em saúde.

\section{Referências:}

1. MINAYO, M. C. de S.; SOUZA, E. R. Violência e saúde como um campo interdisciplinar e de ação coletiva. História, Ciências, Saúde- Manguinhos, IV(3): 513- 


\section{ANAIS DO XIII CONGRESSO MÉDICO ACADÊMICO, 2019; 08-61}

531, nov. 1997-fev. 1998. Disponível em: <http://www.scielo.br/pdf/hcsm/v4n3/v4n3a06>. Acesso 04/02/2019.

2. GOMES, N. P.; DINIZ, N. M. F.; SILVA, C. C. F.; SANTOS, J. N. B. Enfrentamento da violência doméstica contra a mulher a partir da interdisciplinaridade e intersetorialidade. Rev Enferm UERJ. 2009;17(1):14-7. Disponível em: <http://www.facenf.uerj.br/v17n1/v17n1a03.pdf>. Acesso 04/02/2019. 


\title{
PERFIL EPIDEMIOLÓGICO DOS PACIENTES COM NÓDULOS TIREOIDIANOS DO AMBULATÓRIO DE ENDOCRINOLOGIA- TIREOIDE DA UFJF
}

\author{
Paiva ${ }^{4}$; Christianne Toledo Souza Leal ${ }^{5}$. \\ ${ }^{1}$ Acadêmica de Medicina; Universidade Federal de Juiz de Fora; \\ ${ }^{2}$ Acadêmica de Medicina; Universidade Federal de Juiz de Fora; \\ ${ }^{3}$ Acadêmica de Medicina; Universidade Federal de Juiz de Fora; \\ ${ }^{4}$ Acadêmica de Medicina; Universidade Federal de Juiz de Fora; \\ ${ }^{5}$ Médica; Universidade Federal de Juiz de Fora.
}

Camila Rocha Firmo ${ }^{1}$; Franciane Reis ${ }^{2}$; Carolina Teixeira Heleno ${ }^{3}$; Paula Celline Duque

Autor para correspondência:
Camila Rocha Firmino
Email: camilarfirmo@ gmail.com

\section{RESUMO}

Introdução: A elaboração deste estudo resultou da necessidade dos profissionais em identificar o perfil epidemiológico dos pacientes com nódulo(s) de tireoide em acompanhamento no ambulatório de Endocrinologia-tireoide do Hospital Universitário de Juiz de Fora (HU-UFJF) e comparar com dados existentes na literatura. Objetivo: O objetivo principal do estudo é avaliar o perfil epidemiológico e as características preponderantes dos pacientes com nódulo(s) tireoidiano(s) que são acompanhados no serviço de Endocrinologia do HU-UFJF, com a finalidade de nortear o manejo clínico e apresentar dados atualizados gerados a partir desta avaliação. Material e Método: Foram avaliados 117 prontuários do período de janeiro de 2017 a maio de 2018. O estudo avaliou retrospectivamente os prontuários de pacientes com doença nodular da tireoide em ambulatório especializado. Foram excluídos 34 prontuários de pacientes previamente tireoidectomizados e aqueles com informações incompletas sobre número e localização dos nódulos e também sobre fatores de risco para doença tireoidiana. Os dados analisados foram os seguintes: sexo, idade, história familiar de neoplasia da tireóide, meio de diagnóstico do nódulo, número de nódulos, localização e radioterapia prévia da região cervical. Resultados e Discussão: A análise dos 83 prontuários evidenciou que $65,1 \%$ dos pacientes tiveram seu nódulo diagnosticado através de palpação tireoidiana e que estes nódulos acometem majoritariamente mulheres com idade entre 20 e 70 anos. Apenas 19,3\% possuem história familiar positiva para câncer de tireoide. Dentre os prontuários analisados, nenhum dos pacientes foi exposto à radioterapia cervical e apenas $2,4 \%$ foram expostos previamente à radiação ionizante. No total foram encontrados 187 nódulos tireoidianos, 62,7\% eram multifocais e 37,3\% eram unifocais. Em 50,6\% dos pacientes ambos os lobos da tireóide estavam acometidos; $26,5 \%$ apresentavam acometimento apenas no lobo esquerdo; $18,1 \%$ apenas no lobo direito e 4,8\% apresentavam acometimento no istmo. Conclusão: Analisando os dados obtidos é possível concluir que a maioria dos pacientes atendidos no ambulatório Endocrinologia-tireóide da UFJF possuem afecções tireoidianas multinodulares com características de benignidade. Sendo assim, o manejo clínico desses pacientes deve incluir o acompanhamento regular no serviço de Endocrinologia com a realização de ultrassonografia anualmente e dosagem de hormônios tireoidianos semestralmente.

Palavras-chave: Tireoide, Nódulos; Benignidade. 


\section{ANAIS DO XIII CONGRESSO MÉDICO ACADÊMICO, 2019; 08-61}

\section{Referências:}

1- KIMURA, ET et al. Doença Nodular da Tireoide: Diagnóstico. Diretrizes Clínicas na Saúde Suplementar, [S.I.], p. 1-14, jan. 2011. 


\title{
APRENDIZADO MELHORADO POR PROVAS COMO METODOLOGIA DE ESTUDO: UMA REVISÃO NARRATIVA
}

\author{
Tomás Gazzinelli Marçal ${ }^{1}$; Aline Cristina $\mathrm{Mafra}^{2}$; Victoria Barreto de Araújo Porto ${ }^{3}$; Alice \\ Belleigoli Rezende ${ }^{4}$ \\ ${ }^{1}$ Acadêmico de Medicina; Universidade Federal de Juiz de Fora \\ ${ }^{2}$ Acadêmica de Medicina; Universidade Federal de Juiz de Fora \\ ${ }^{3}$ Acadêmica de Medicina; Universidade Federal de Juiz de Fora. \\ ${ }^{4}$ Docente; Faculdade de Medicina da Universidade Federal de Juiz de Fora
}

\author{
Autor para correspondência: \\ Tomás Gazzinelli Marçal \\ Email: tomas.gazzinelli@medicina.ufjf.br
}

\section{RESUMO}

Introdução e objetivos: Apesar do crescente número de publicações enfocando a eficácia do "aprendizado melhorado por provas", sua utilização é rara pelos estudantes, além de existirem poucos trabalhos com essa temática em português $\mathbf{1 , 2 , 3 , 4 , 5 , 6 , 7}$. Nesse contexto, essa revisão busca preencher a lacuna existente e aproximar esse recurso da realidade do ensino brasileiro, através da compreensão desta técnica de estudo e da comparação com as outras estratégias utilizadas pelos alunos. Metodologia: Foi feita uma pesquisa na bibliografia completa de 5 autores de índice-H maior que 27 na área de psicologia cognitiva do aprendizado (Roediger HL, Karpicke JD, Dunlosky J, Butler AC e Marsh EJ), pelo Google Scholar. Além disso, foi empregada uma equação booleana com descritores MeSH e nomes de outros autores, na base de dados PubMed. Essa pesquisa resultou em mais 321 artigos, dos quais foram, então, selecionados, a partir da leitura de títulos e abstratos, levando a um total de 27 artigos, os quais passaram por análise dos autores. Resultados e discussão: Podemos destacar que a metodologia de estudo mais eficaz, tendo como objetivo o aprendizado a longo prazo, é a formulação e resposta de perguntas $\mathbf{8 , 9 , 1 0 , 1 1 , 1 2 , 1 3 , 1 4 , 1 5}$. Diversos estudos mostraram, ainda, que reestudar a mesma passagem diversas vezes, não aumenta significativamente a retenção, ao contrário de responder perguntas repetidas vezes ${ }^{\mathbf{1 2 , 1 5}}$. Os períodos entre as sessões de estudo são de suma importância para aumentar a retenção ${ }^{\mathbf{1 6 , 1 7 , 1 8}}$. Nesse contexto, vale ressaltar que os intervalos progressivos entre as sessões de estudo são melhores para o aprendizado no curto prazo, enquanto intervalos de mesma duração favorecem a retenção a longo prazo, mas há controvérsias ${ }^{\mathbf{8 , 1 7}, 19}$. Ainda, intercalar os assuntos têm maior efetividade e acumular grandes quantidades de conteúdo diminui a eficiência do estudo ${ }^{\mathbf{1 2 , 1 8 , 2 0}}$. Partindo do pressuposto que responder perguntas repetidas vezes é a melhor prática de estudo, é necessário enfatizar a importância do feedback. Esse melhora a retenção, corrige erros no aprendizado e aumenta o entendimento do assunto $\mathbf{1 , 4 , 5 , 8 , 2 1 , 2 2 , 2 3}$. Porém, esse método deve explicar o porquê de uma determinada resposta ser a correta, para, efetivamente, ter maior eficácia ${ }^{\mathbf{2 2 , 2 4}}$. Diversos trabalhos demonstraram o desconhecimento dos acadêmicos em relação à essa prática de estudos ${ }^{12,25,26,27}$. A maioria dos estudantes acredita que reler, sublinhar e fazer mapas conceituais leva a maior aprendizagem, apesar desses métodos serem os que apresentam menor eficiência 11,16,19. Ademais, vale ressaltar que responder perguntas não demora mais tempo que reler o tema, e que formular as questões a serem respondidas demora de duas a três vezes mais que respondê-las, porém não há diferença em performance entre elaborar as perguntas e respondê-las ou somente respondê-las ${ }^{12,15}$. Conclusão: $O$ uso de perguntas e respostas é uma metodologia pouco utilizada para o aprendizado no meio acadêmico e sua implementação no dia a dia pode aumentar muito a qualidade de assimilação dos alunos. Portanto, é necessário que os estudantes compreendam como esquematizar o estudo com 
maior eficiência, antes de começarem a aprender. Além disso, devido à escassez de publicações brasileiras, são necessárias maiores pesquisas sobre essa temática no país.

Palavras-chave: Educação; Aprendizado; Testes; Eficiência

\section{Referências:}

1. SPITZER, Hebert F. Studies in Retention. The Jornal of Educational Psychology, Iowa, v.30, n. 9, p. 641-656, dez. 1939. Disponível em: <https://psycnet.apa.org/record/194002338-001>. Acesso em: fev, 2019.

2. KROMANN, Charles Boy; JENSEN, Morten L; RINGSTED, Charlotte. The effect of testing on skills learning. Medical Education, Copenhague, v.43, n.1, p.21-27, jan. 2009. Disponível em: <https://www.ncbi.nlm.nih.gov/pubmed/19140995>. Acesso em: fev, 2019.

3. WHELEER, Mark; EWERS, Michael; BUONANNO, Joseph. Different rates of forgetting following study versus test trials. Memory, Filadélfia, v.11, n.6, p. 571-580, nov. 2003. Disponível em: <https://www.ncbi.nlm.nih.gov/pubmed/14982124>. Acesso em: fev,2019.

4. LARSEN, Douglas P.; BUTLER Andrew C; ROEDIGER Henry L. III. Test-enhanced Learning. Medical Education, Missouri, v.42, n.10, p. 959-966, out. 2008. Disponível em: <https://www.ncbi.nlm.nih.gov/pubmed/18823514>. Acesso em: fev, 2019.

5. LARSEN, Douglas P.; BUTLER Andrew C; ROEDIGER Henry L. III. Repeated testing improves long-term retention relative to repeated study : a randomized controlled trial . Medical Education, Missouri, v.43, n.12, p. 1174-1181, dec. 2009. Disponível em: 〈https://www.ncbi.nlm.nih.gov/pubmed/19930508>. Acesso em: fev,2019.

6. ROEDIGER Henry L. III; KARPICKE Jeffrey D. Test-Enhanced Learning: Taking Memory Tests Improves Long-Term Retention. Psychological Science, Nova York, v.17, n.3, p. 249-255, mar. 2006. Disponível em: <https://www.ncbi.nlm.nih.gov/pubmed/16507066>. Acesso em: fev,2019.

7. BINKS, Sally. Testing enhances learning: A review of the literature. Journal of Professional Nursing, Amsterdã, v. 34, n.3, p. 205-2010, mai. 2018. Disponível em: <https://psycnet.apa.org/record/2018-30170-015>. Acesso em: fev,2019.

8. ROEDIGER Henry L. III; BUTLER Andrew C. The Critical Role of Retrieval Practice in Long-Term Retention. Trends in Cognitive Sciences. Amesterdã, v.15, n. 1, p.2027, jan. 2011. Disponível em: <https://www.ncbi.nlm.nih.gov/pubmed/20951630>. Acesso em: fev,2019.

9. LARSEN, Douglas $P$ et al. The effects of test-enhanced learning on long-term retention . Neurology. Filadélfia, v. 84, n.7, p. 748-754, fev. 2015. Disponível em: <https://www.ncbi.nlm.nih.gov/pubmed/25609761>. Acesso em: fev,2019.

10. KARPICKE, J. D. Retrieval-based learning: A decade of progress. Cognitive psychology of memory, Oxford, v.2, n.1, p. 487-514, jan. 2017. Disponível em: <https://www.ncbi.nlm.nih.gov/pmc/articles/PMC4786565/>. Acesso em: fev,2019. 
11. KARPICKE, J. D.; BLUNT, J. R. Retrieval practice produces more learning than elaborative studying with concept mapping. Science, Nova York, n. 331, p. 772-775, fev. 2011. Disponível em: <https://www.ncbi.nlm.nih.gov/pubmed/21252317>. Acesso em: fev, 2019.

12. DUNLOSKY, John et al . Improving Students' Learning With Effective Learning Techniques: Promising Directions From Cognitive and Educational Psychology. Psychological Science, Nova York, v.14, n.1, p. 4-58, jan. 2013. Disponível em: <https://psycnet.apa.org/record/2013-00960-002>. Acesso em: fev,2019.

13. SMITH, MA ; KARPICKE, J. D Retrieval practice with short-answer, multiplechoice, and hybrid tests. Memory, Filadélfia, v. 22, n. 7, p. 784-802, jan. 2014. Disponível em: <https://www.ncbi.nlm.nih.gov/pubmed/24059563>. Acesso em: fev,2019.

14. RAWSON, K. A. ; DUNLOSKY, J. ; \& SCIARTELLI S. M. The power of successive relearning: Improving performance on course exams and long-term retention. Educational Psychology Review, Nova York, v. 25, n.4, p. 523-548, set. 2013. Disponível em: <https://psycnet.apa.org/record/2013-34083-001>. Acesso em: fev,2019.

15. WEISTEIN,Y; MCMERMOTT, KB; ROEDIGER Henry L. III A comparison of study strategies for passages: Rereading,answering questions, and generating questions. Journal of Experimental Psychology:Learning, Memory, and Cognition, Nova York, v.16, n.3, p.308-316, mar 2010. Disponível em: <https://eric.ed.gov/?id=EJ899083>. Acesso em: fev, 2019.

16. KARPICKE, J. D . A powerful way to improve learning and memory. Psychological Science, Nova York. Disponível em: <https://www.apa.org/science/about/psa/2016/06/learning-memory>. Acesso em: fev, 2019.

17. BUTLER, A. C. ; KARPICKE, J. D; ROEDIGER Henry L. III. The effect of type and timing of feedback on learning from multiple-choice tests. Journal of Experimental Psychology: Learning, Memory, and Cognition, Nova York, v.13, n. 4, p. 273-281, dez 2007. Disponível em: <https://www.ncbi.nlm.nih.gov/pubmed/18194050>. Acesso em: fev,2019.

18. MEREHEAD, $\mathrm{K}$ et al. Does mediator use contribute to the spacing effect for cued recall? Critical tests of the mediator hypothesis. Memory, Filadélfia, v. 26, n.4, p. 535-546, Abr 2018. Disponível em: <https://www.ncbi.nlm.nih.gov/pubmed/28978279>. Acesso em: fev, 2019.

19. KARPICKE, J. D; BAUERNSCHMIDT A. Spaced retrieval: absolute spacing enhances learning regardless of relative spacing. Journal of Experimental Psychology: Learning, Memory, and Cognition, Nova York, v.37, n.5, p. 1250-1257, jan. 2011. Disponível em: <https://www.ncbi.nlm.nih.gov/pubmed/21574747>. Acesso em:fev, 2019.

20. WAHLHEIM, C. N. ; DUNLOSKY, J. ; Jacoby, L. L. Spacing enhances the learning of natural concepts:an investigation of mechanisms, metacognition, and aging. Mem Cognit, Nova York, v. 3, n.5, p. 750-763, jul 2011. Disponível em: <https://www.ncbi.nlm.nih.gov/pmc/articles/PMC3085105/>. Acesso em: fev, 2019. 
21. LARSEN, D. P. et al. The effects of test-enhanced learning on long-term retention in AAN annual meeting courses. Neurology., Filadélfia, v.84, n. 7, p.748-54, fev 2015. Disponível em: <https://www.ncbi.nlm.nih.gov/pubmed/25609761/>. Acesso em: fev, 2019.

22. FAZIO, L.K. et al. Receiving right/wrong feedback: consequences for learning. Memory , Filadélfia, v. 18, n.3, p. 335-50, Abr 2010. Disponível em: <https://www.ncbi.nlm.nih.gov/pubmed/20408043>. Acesso em:fev, 2019.

23. SUMERACKI, M. A. ; KARPICKE, J. D .Retrieval practice with short-answer, multiplechoice, and hybrid tests. Memory, Filadélfia, v.22, n. 7, p 784-802, jun 2013. Disponível em: <https://www.ncbi.nlm.nih.gov/pubmed/24059563>. Acesso em: fev,2019.

24. BLUTER, A.C. ; GODBOLE, N.; MARSH, E. J. Explanation Feedback Is Better Than Correct Answer Feedback for Promoting Transfer of Learning. Journal of Educational Psychology, Iowa, v.105, n.12, p. $290-298$, jan 2013. Disponível em:<https://marshlab.psych.duke.edu/publications/ButlerGodbole\&Marsh2013_.EdPsych.pdf $>$. Acesso em: fev, 2019.

25. ROEDIGER Henry L. III; KARPICKE Jeffrey D.The Power of Testing Memory: Basic Research and Implications for Educational Practice. Psychological Science, Nova York, v.1, n.3, p. 181-210, set 2006. Disponível em: <https://journals.sagepub.com/doi/10.1111/j.17456916.2006.00012.x>. Acesso em: fev, 2019.

26. KARPICKE, J. D Retrieval-Based Learning: Active Retrieval Promotes Meaningful Learning. Psychological Science, Nova York, v.21, n.3, p. 157-163, mar 2012. Disponível em: <https://psycnet.apa.org/record/2012-14871-002>. Acesso em: fev, 2019.

27. GRIMALDI, P.J. ; KARPICKE, J. D; Guided retrieval practice of educational materials using automated scoring. Journal of Educational Psychology, Iowa, v.106, n.1, p. 58-68, jan 2014. Disponível em: <https://psycnet.apa.org/record/2013-22184-001>. Acesso em: fev, 2019. 


\title{
RASTREAMENTO ORGANIZADO DOS CÂNCERES DE MAMA E COLO DO ÚTERO EM JUIZ DE FORA-MG: IMPLANTAÇÃO DE UM PROJETO PILOTO - UM RELATO DE EXPERIÊNCIA
}

\author{
Yara Mendes Silva ${ }^{1}$; Iara Carlin Torres ${ }^{2}$; Rafaella Angélica Vieira e Silva ${ }^{3}$; Isabela de \\ Oliveira Araújo ${ }^{4}$; Jonas Munck de Oliveira ${ }^{5}$; Maria Teresa Bustamante Teixeira ${ }^{6}$. \\ ${ }^{1}$ Acadêmica de Medicina; Universidade Federal de Juiz de Fora; \\ ${ }^{2}$ Acadêmica de Medicina; Universidade Federal de Juiz de Fora; \\ ${ }^{3}$ Acadêmica de Medicina; Universidade Federal de Juiz de Fora; \\ ${ }^{4}$ Acadêmica de Medicina; Universidade Federal de Juiz de Fora; \\ ${ }^{5}$ Acadêmica de Medicina; Universidade Federal de Juiz de Fora; \\ ${ }^{6}$ Docente; Faculdade de Medicina da Universidade Federal de Juiz de Fora.
}

\author{
Autor para correspondência: \\ Yara Mendes Silva \\ Email: yara_mendes.s@hotmail.com
}

\section{RESUMO}

Introdução: No Brasil, os cânceres de mama e do colo de útero destacam-se como problemas de saúde pública, desta forma, o controle dessas neoplasias é considerado uma prioridade nacional. ${ }^{1}$ A mamografia de rastreio, segundo o Ministério da Saúde, deve ser realizada a cada dois anos, em mulheres assintomáticas, na faixa etária de 50 a 69 anos. Já o rastreamento citopatológico é recomendado para mulheres de 25 a 64 anos, a cada três anos após dois exames com resultados negativos realizados anualmente. ${ }^{2}$ Porém, ainda são realizados de forma oportunística no país, que além de ser menos efetiva no impacto sobre a mortalidade, é mais onerosa para o sistema de saúde. ${ }^{3}$ Objetivos: Implantar um programa piloto de rastreamento organizado dos cânceres de mama e colo do útero em área adscrita por Unidade de Atenção Primária à Saúde (UAPS) no município de Juiz de Fora. Será criado e alimentado um banco de dados contendo informações de todas as mulheres atendidas pela UAPS que estão na faixa etária preconizada para o rastreamento citopatológico e mamográfico e assim, melhorar a cobertura do rastreio. Além disso, promover a educação em saúde e a educação permanente com foco na prevenção dos cânceres, inserir os acadêmicos da área da saúde na atenção primária desde o início de sua formação e promover, através do tripé ensino, pesquisa e extensão, a aproximação da academia com os serviços de saúde e a população. Relato de Experiência: A extensão está sendo realizada nas UAPS Bairro Industrial e o grupo é composto por acadêmicos do curso de medicina, pela equipe de saúde da UAPS, por alunos do Programa de Pós-graduação em Saúde Coletiva da UFJF, por docentes e pelos coordenadores do projeto. Nas etapas iniciais, o grupo se reuniu para elaborar duas cartilhas que eram direcionadas, principalmente para a equipe de saúde da UAPS, a fim de contribuir na divulgação de conhecimentos científicos, contendo informações dos dois tipos de cânceres pesquisados. Foram então realizadas reuniões com toda a equipe da UAPS e da UFJF, para a discussão dos temas e levantamento de propostas para a implantação do rastreamento organizado. Em seguida, o grupo da UFJF se reuniu para confeccionar materiais a serem utilizados nas salas de espera, momentos em que os alunos irão interagir com as mulheres usuárias da UAPS para transmitir informações importantes sobre os cânceres, com enfoque na prevenção e no rastreamento correto. A próxima etapa será o levantamento da população feminina alvo, cadastrada na UAPS, para câncer de mama e do colo do útero, pela equipe do projeto junto aos agentes comunitários. Estes dados serão analisados com o objetivo de, inicialmente, realizar o diagnóstico da situação e, posteriormente, monitorar a situação de rastreamento de cada mulher. Resultados e Discussão: Até o momento, as ações realizadas foram bem-sucedidas. O contato com os profissionais de saúde que trabalham na UAPS foi 
esclarecedor e todos aderiram ao projeto. Conclusão: Por meio da extensão, os acadêmicos podem contribuir com a implantação de um serviço organizado de rastreio dos cânceres de mama e do colo do útero, a fim de diminuir os custos com o rastreamento e consequentemente a mortalidade pela doença. Ademais, a aproximação dos acadêmicos com os profissionais e usuários dos serviços públicos de saúde se prova, a cada etapa, um método eficaz de aprendizado teórico-prático.

Palavras-chave: Saúde da Família, UAPS, Câncer de Mama, Câncer do Colo do Útero, Extensão.

\section{Referências:}

1. AZEVEDO E SILVA G. et al. A situação dos cânceres do colo do útero e da mama no Brasil. Rio de Janeiro: Outras Letras; 2015.

2. BRASIL. MINISTÉRIO DA SAÚDE. INSTITUTO NACIONAL DO CÂNCER JOSÉ DE ALENCAR GOMES DA SILVA. Diretrizes para a detecção precoce do câncer de mama no Brasil. Rio de Janeiro: Instituto Nacional de Câncer José Alencar Gomes da Silva; 2015.

3. BRASIL. MINISTÉRIO DA SAÚDE. SECRETARIA DE ATENÇÃO À SAÚDE. DEPARTAMENTO DE ATENÇÃO BÁSICA. Rastreamento. Brasília: Ministério da Saúde; 2010. 


\title{
RELATO DE EXPERIÊNCIA: COMUNIDADE SAUDÁVEL - PROMOVENDO SAÚDE E REDUZINDO VULNERABILIDADES ATRAVÉS DO ACESSO E INTERSETORIALIDADE
}

\author{
Ana Luísa Soares Costa1; Gabriela Ferreira da Silva²; Francieli do Carmo Pereira Oliveira ${ }^{3}$; \\ Márcio José Martins Alves ${ }^{4}$. \\ ${ }^{1}$ Acadêmica de Medicina; Universidade Federal de Juiz de Fora; \\ ${ }^{2}$ Acadêmica de Medicina; Universidade Federal de Juiz de Fora; \\ ${ }^{3}$ Acadêmica de Medicina; Universidade Federal de Juiz de Fora; \\ ${ }^{4}$ Docente; Faculdade de Medicina da Universidade Federal de Juiz de Fora.
}

Autor para correspondência: Ana Luísa Soares Costa;

Email: costa.anasoares@gmail.com

\section{RESUMO}

Introdução: A promoção de saúde nas comunidades é uma das ações fundamentais quando se visa atuar sobre as vulnerabilidades sociais. Para facilitar o acesso à saúde é preciso antes conhecer o território da comunidade, tanto físico quanto socioeconômico e cultural, a fim de se estabelecer um vínculo. Nesse sentido, o Projeto "Comunidade Saudável" busca ampliar o acesso aos serviços de saúde, atuando em conjunto com ONGs, Instituições Governamentais, Universidade, Serviços de Saúde, lideranças comunitárias e todos os demais integrantes da comunidade do bairro Dom Bosco, local de grande vulnerabilidade localizada no entorno da UFJF. O projeto é baseado na premissa da importância da intersetorialidade para o acompanhamento integral da população local ${ }^{1}$. Objetivos: O presente trabalho objetiva relatar a experiência do projeto de extensão "Comunidade Saudável", que através de uma rede assistencial e intersetorial, presta atendimento médico para os jovens de 7 a 14 anos assistidos pelo Instituto Profissional Dom Orione (Instituição religiosa prestadora do Serviço de Convivência e Fortalecimento de Vínculo para o bairro Dom Bosco ${ }^{2}$ ) e suas famílias, além de realizar ações socioeducativas de promoção à saúde. Relato de experiência: A atuação do projeto se dá por discentes da Faculdade de Medicina da UFJF, que, orientadas pelo docente responsável, se distribuem em atividades que se referem a dois eixos principais: a promoção do acesso, por meio da marcação e acompanhamento de consultas quinzenais na Unidade Básica de Saúde do Dom Bosco e a educação em saúde, promovendo e elaborando atividades lúdicas e educativas sobre temas como higiene e limpeza pessoal, sexualidade e violência, no espaço do Instituto Dom Orione. Resultados e Discussão: Diante das barreiras organizacionais e socioculturais observadas, o projeto têm facilitado o acesso desses jovens e de seus familiares ao serviço de saúde, transformando a UBS Dom Bosco em sua porta de entrada, já que a unidade ainda não possui Estratégia de Saúde da Família. Há, ainda, o contato com ONGs atuantes no bairro, ampliando as inserções na comunidade, e a participação em reuniões mensais da rede CRAS Centro, espaço de gestão dos programas e abordagens socioassistenciais individualizadas. Conclusão: A elaboração dos planos de cuidado para famílias em risco social, através do trabalho intersetorial, propicia aos integrantes do projeto compreender as complexas interações que atuam nas condições de vida e saúde dessas pessoas. Objetivando a superação da fragmentação do conhecimento e das estruturas sociais, a intersetorialidade surge como uma nova forma de trabalhar, governar e construir políticas públicas. Espera-se assim produzir efeitos mais significativos na resolução dos problemas e necessidades da comunidade local.

Palavras-chave: promoção de saúde, intersetorialidade, redes assistenciais 


\section{Referências:}

1- DIAS, Maria Socorro de Araújo et al. Intersetorialidade e Estratégia Saúde da Família: tudo ou quase nada a ver?. Ciência \& Saúde Coletiva, v. 19, p. 4371-4382.

2- Serviço de Proteção e Atendimento Integral à Família e Serviço de Convivência e Fortalecimento de Vínculos. Articulação necessária na Proteção Social Básica. Disponível em:

http://www.mds.gov.br/webarquivos/arquivo/assistencia_social/cartilha_paif_2511.pdf. Acesso em: 20 de março de 2019. 


\title{
PERDENDO O MEDO DO MÉDICO COM O PROJETO DE EXTENSAO HOSPITAL DE URSINHOS: RELATO DE EXPERIÊNCIA
}

\author{
Vivian Reis Ferrarez ${ }^{1}$; Ana Teresa Dias Albino Destro de Macêdo ${ }^{1}$; Angela Caroline Dias \\ Albino Destro de Macêdo ${ }^{1}$; Eduardo Lacerda de Castro Lobo ${ }^{1}$; Everton Soares Rocha ${ }^{2}$. \\ ${ }^{1}$ Acadêmico de Medicina da Universidade Federal de Juiz de Fora. \\ ${ }^{2}$ Docente de Medicina da Universidade Federal de Juiz de Fora.
}

\author{
Autor para Correspondência: \\ Vivian Reis Ferrarez \\ Email: vivian_ferrarez@hotmail.com
}

\section{RESUMO}

Introdução: $\mathrm{O}$ adoecimento é um desvio da rotina natural da criança, sendo constantemente vinculado a sentimentos de medo, angústia e insegurança pelo paciente. Realizado por estudantes de medicina em parceria com escolas de educação Infantil e creches, o projeto trabalha numa reconstrução do ideário infantil a respeito do médico e da instituição hospitalar ainda nas fases iniciais da vida, utilizando para isso o lúdico, de forma a evitar que novas gerações cresçam com uma visão ameaçadora e desenvolvam fobias a cerca dos centros e procedimentos de saúde. Objetivos: O intuito do projeto é fazer com que as crianças percam o medo dos médicos, de procedimentos e do ambiente hospitalar, através de uma atividade lúdica. Ao levar seu ursinho para realizar procedimentos como vacinação e raio x, a criança transfere seus medos e angustias ao brinquedo e se vê na posição de um responsável que preza pela saúde do filho. Com isso, ela compreende a importância de cuidar da saúde e entende que não há razões para temer qualquer profissional da saúde. $\mathrm{O}$ projeto ainda proporciona a humanização dos estudantes de medicina, já que eles possuem um contato direto com as crianças. Relato de experiência: A estrutura do "Hospital de Ursinhos" é composta de setores que simulam o ambiente hospitalar, dentre eles: recepção, farmácia, consultórios e salas de vacinação, de cirurgia, de exame de imagem e de curativos. Os acadêmicos explicam às crianças como funciona o hospital $\mathrm{e}$ as crianças são convidadas a escolherem seus ursinhos e imaginarem um agravo em saúde para eles. Os acadêmicos "atendem" os ursinhos de pelúcia acompanhado das crianças, de 3 a 8 anos. As crianças circulam por toda a estrutura enquanto aprendem ludicamente a importância do cuidado com a saúde. É trabalhada educação em saúde, prevenção de doenças, importância da vacinação, adesão ao tratamento, conhecimento dos procedimentos e aparatos médicos. Discussão e Resultados: Visto que o adoecimento é associado a sentimentos de vulnerabilidade, é comum observarmos grande resistência da população a submeter-se a atendimentos e procedimentos de saúde, tornando-se fator de risco para a saúde e desenvolvimento integral da criança. Visto a importância do brincar na construção do psiquismo, desenvolvimento físico, emocional, sensorial e autoconsciência, o projeto busca estimular o autocuidado. Esperamos que as crianças se familiarizem com ambientes, atendimentos, profissionais e procedimentos de saúde, mas também se divirtam durante a sua realização. Além disso, esperamos que os estudantes de medicina desenvolvam habilidades no contato com a faixa etária pediátrica e suas peculiaridades, e assim, desenvolvam uma boa relação médico-paciente. Conclusão: Ressalta-se a importância do projeto no enfrentamento da iatrofobia ao gerar a familiaridade da criança, através da ludicidade, com o ambiente hospitalar. Assim, após as ações, feedbacks das instituições sobre a mudança comportamental das crianças, nas instituições e no ambiente familiar, foram expressivas em relação as práticas do auto cuidado. Além das crianças, os relatos dos estudantes de medicina que participaram da ação foram positivos em relação ao desenvolvimento humano, fator que, segundo eles, contribuiu para o maior facilidade de estabelecimento da relação médico-paciente, principalmente com o público infantil. 
Palavras- chave: Hospital de Ursinhos, Lúdica, Humanização.

\section{Referências:}

1- MENDES, M V. O brincar como promoção da saúde: a brinquedoteca em uma UBS. Pontifícia Universidade Católica Faculdade de Psicologia. São Paulo, 2008.

2- Winnicott, D. W. (1971/1975). O brincar e a realidade. Trad. José Octavio de Aguiar Abreu e Vanede Nobre. Rio de Janeiro: Imago.

3- PELICIONI, Maria C. F; TORRES, A. L; Escola promotora da saúde. Universidade de São Paulo. Saúde Publica: São Paulo, 1999. 


\title{
RELATO DE EXPERIÊNCIA: IMPORTÂNCIA DO CONTATO PRECOCE DO ESTUDANTE DE MEDICINA COM O AMBIENTE MÉDICO
}

\begin{abstract}
Ana Luíza de Castro Carvalho ${ }^{1}$; Gabrielle de Moura Lopes ${ }^{2}$; Giovanna Alves de Oliveira ${ }^{2}$; Giulia Machado Caldeira Ardisson ${ }^{2}$; Everton Soares Rocha ${ }^{3}$; Valquíria Pereira de Medeiros ${ }^{4}$.

1 Acadêmicas pela Universidade Federal de Juiz de Fora/ Faculdade de Medicina;

${ }^{2}$ Graduação em medicina pela Universidade Federal de Juiz de Fora/ Faculdade de Medicina; especialização em pediatria e puericultura pela Pontifícia Universidade Católica do Rio de Janeiro; residência médica em pediatria pela policlínica de Botafogo Rio de Janeiro; mestre em saúde pela Universidade Federal de Juiz de

Fora; departamento de Bioquímica, Instituto de Ciências Biológicas- UFJF.

${ }^{3}$ Graduação em biologia pela Universidade Federal do Rio Grande do Norte/ Faculdade de Ciências Biológicas; mestrado em Bioquímica pela Universidade Federal do Rio Grande do Norte; doutorado em biologia molecular pela Universidade Federal de São Paulo; docente Departamento de Bioquímica, Instituto de Ciências BiológicasUFJF.
\end{abstract}

Autor para Correspondência:

Ana Luíza de Castro Carvalho Email: aluizac1255@hotmail.com

\section{RESUMO}

Introdução e Objetivos: Estudos mostram que existe uma correlação entre um atendimento médico satisfatório e a melhora do estado de saúde dos pacientes ${ }^{1}$, sendo relatado que os pacientes se beneficiam mais dos médicos e estudantes de medicina, quando estes se empenham na consulta ${ }^{2}$. Tendo em vista esses estudos realizados e, baseado no fato de que na faculdade de medicina da Universidade Federal de Juiz de Fora o aluno tem seu primeiro contato com pacientes no quarto período, foi criado o projeto de extensão "Médico por um dia". Esse projeto foi pensado e desenvolvido com o pressuposto de estimular e possibilitar aos estudantes recém aprovados no curso de medicina o contato com o ambiente médicohospitalar. Dessa forma, há um auxílio em sua formação, bem como na humanização do cuidado com o paciente no futuro, além do desenvolvimento da capacidade de ter empatia. $\mathrm{O}$ objetivo desse projeto é despertar o interesse do acadêmico de medicina desde o primeiro período quanto à percepção da importância de uma visão mais humanizada da relação médico-paciente, e buscar sua construção. Relato de Experiência: O projeto conta com a participação de trinta alunos do primeiro período do curso de medicina selecionados por sorteio. Ocorre uma visita, guiada por preceptores, ao Hospital Universitário (HU)- Unidade Santa Catarina, a qual recebe o apoio da Doutora Ângela Maria Gollner e do Professor Sérgio Antonio Ribeiro Campos, ambos membros da Gestão de Ensino e Pesquisa do HU. Em um segundo momento, é realizada uma sala de espera pelos alunos, no HU- Unidade Dom Bosco, com a abordagem de temas médicos relevantes para a comunidade e interação entre alunos e pacientes. Resultados: Os alunos tiveram contato precoce com os pacientes na sala de espera, onde houve uma relação de transferência e contratransferência entre estudantes e pacientes, além de um incremento de conhecimento de ambas as partes. Discussão: A boa comunicação médico-paciente tem o potencial de ajudar a regular as emoções dos pacientes, facilitar a compreensão de informações médicas, além de permitir melhor identificação das necessidades, percepções e expectativas dos pacientes ${ }^{3}$. Tendo isso em vista, o projeto "Médico por um dia" corrobora com a formação de médicos capazes de estabelecerem uma efetiva interação com seus pacientes. Conclusão: Em suma, a realização do projeto "Médico por um dia" criou uma possibilidade para que estudantes tivessem um contato precoce com pacientes, e com essa vivência, iniciassem o aprendizado prático acerca da grande importância que existe no desenvolvimento de uma boa relação médico-paciente. 


\section{ANAIS DO XIII CONGRESSO MÉDICO ACADÊMICO, 2019; 08-61}

Palavras-chave: Graduandos de Medicina. Relação médico-paciente. Comunicação.

\section{Referências:}

1- SUCUPIRA, Ana Cecília. A importância do ensino da relação médico-paciente e das habilidades de comunicação na formação do profissional de saúde. Interface (Botucatu), Botucatu, v. 11, n. 23, p. 624-627, Dez. 2007. Disponível em: $<$ http://www.scielo.br/scielo.php?script=sci_arttext\&pid=S141432832007000300016\&lng=en\&nrm=iso>. Acesso em: 20 mar. 2019.

2- KIYOHARA, Leandro Yoshinobu et al. The patient-physician interactions as seen by undergraduate medical students. São Paulo Med. J., São Paulo, v. 119, n. 3, p. 97-100, May 2001.

Disponível

em: <http://www.scielo.br/scielo.php?script=sci_arttext\&pid=S1516-

$31802001000300002 \& \operatorname{lng}=$ en\&nrm=iso>. Acesso em: 18 fev. 2019.

3- HA, Jennifer Fong; LONGNECKER, Nancy. Doctor-Patient Communication: A Review. The Ochsner Journal, New Orleans, v.10, n.1., p. 38-43, Mar. 2010. Disponível em: < https://www.ncbi.nlm.nih.gov/pmc/articles/PMC3096184/\#!po=1.19048>. Acesso em: 23 Mar. 2019. 


\title{
ABORDAGEM TERAPÊUTICA DA CRIANÇA COM TRANSTORNO DO ESPECTRO AUTISTA: ATENDIMENTO AMPLIADO AOS PAIS
}

\author{
Julia de Araujo Louvain Viana ${ }^{1}$; Lucas Mizrahy Lima²; Márcia Helena Fávero de Souza ${ }^{3}$; \\ Cacilda Andrade de Sá ${ }^{4}$ \\ ${ }^{1}$ Graduanda em Medicina; Universidade Federal de Juiz de Fora (UFJF) \\ ${ }^{2}$ Graduando em Medicina; Universidade Federal de Juiz de Fora (UFJF) \\ ${ }^{3}$ Psiquiatra da Infância e Adolescência, Doutora em Saúde (UFJF), Professora da Faculdade de Medicina da \\ UFJF \\ ${ }^{4}$ Psicóloga, Doutora em Saúde (UFJF), Professora da Faculdade de Medicina da UFJF.
}

Autor para correspondência:

Julia de Araujo Louvain Viana

Email: louvainjulia@gmail.com

\section{RESUMO}

Introdução e Objetivos: Embora seja frustrante e desafiador para muitas famílias receber o diagnóstico de Transtorno do Espectro Autista (TEA) de sua criança, a abordagem multidisciplinar precoce que envolva também os pais nesse processo terapêutico vem se mostrando como uma estratégia importante ${ }^{1}$. Os pais têm a possibilidade de esclarecer suas dúvidas e entender melhor as particularidades do desenvolvimento de seu filho, ao mesmo tempo em que podem aplicar esses conhecimentos na prática, no ambiente doméstico, dando à criança a oportunidade do cuidado integral. Diante deste contexto, o serviço de Psiquiatria e Psicologia Médica do Hospital Universitário da Universidade Federal de Juiz de Fora (HUUFJF) oferece um espaço de psicoterapia para crianças portadoras de TEA. Desta forma, o objetivo do presente trabalho é relatar a experiência desse espaço durante o segundo semestre de 2018. Relato de Experiência: O atendimento foi realizado por uma residente de Psicologia e um residente de Psiquiatria Infantil, vinculados ao HU-UFJF, e por cinco alunos dos cursos de Medicina e Psicologia da UFJF filiados à Liga Acadêmica de Saúde Mental da UFJF, todos orientados pela professora responsável. Às quartas-feiras, pela manhã, as crianças compareciam ao Hospital acompanhadas pelos pais e a equipe se dividia: parte ficava responsável pelo atendimento individualizado à criança e parte oferecia suporte aos pais em outro ambiente. Cada criança era sempre assistida pelos mesmos integrantes da equipe, o que permitiu o estabelecimento de um plano terapêutico individualizado e longitudinal baseado nas suas necessidades específicas; para tal, eram utilizados brinquedos de cunho pedagógico, atividades que estimulavam leitura, a minimização de comportamentos estereotipados, o manejo de frustrações, a interação social e a expressão verbal e corporal. Já o atendimento aos pais dava-se por meio de escuta qualificada, na qual havia espaço para esclarecimento de dúvidas sobre o tratamento de seu filho e sobre o TEA, exposição de inseguranças, frustrações e percepções sobre o contexto familiar atual, e, de acordo com a necessidade, ocorria o encaminhamento para outros profissionais de saúde. Resultados e Discussão: Pôde-se notar, ao longo do período, o estabelecimento de um vínculo por parte das crianças com os membros da equipe que realizavam seu atendimento e visível melhora em muitos dos pontos trabalhados com elas. Observou-se, ainda, o reforço do vínculo dos pais com os próprios filhos e com o serviço, facilitando a apresentação das dificuldades, troca de experiências e conhecimento. Além disso, para a formação médica, a experiência foi relevante na medida em que explicitou a efetividade e a necessidade da interdisciplinaridade na condução dos casos. Foi possível, especialmente para os alunos do curso de medicina, a preparação para conduzir um atendimento médico de qualquer área levando em conta as particularidades e a diversidade do TEA. Também puderam aprender a lidar com as próprias frustrações diante da terapêutica de um quadro crônico para conseguir atender às demandas da família da forma 


\section{ANAIS DO XIII CONGRESSO MÉDICO ACADÊMICO, 2019; 08-61}

mais honesta possível. Conclusão: Ficou clara a evolução dos pacientes em função da metodologia terapêutica aplicada, a criação de vínculos da equipe com toda a família e o engrandecimento teórico-prático por parte dos alunos e profissionais envolvidos no projeto.

Palavras-chave: Transtorno do espectro autista. Pais. Interdisciplinaridade.

\section{Referências:}

1- PICHINI, Fernanda dos Santos et al . Percepção da família e do terapeuta sobre a evolução de crianças em uma abordagem interdisciplinar de intervenção precoce. Rev. CEFAC, São Paulo, v. 18, n. 1, p. 55-66, fev. 2016. Disponível em: $<$ http://www.scielo.br/scielo.php?sc ript=sci_arttext\&pid=S1516-184620160001000 55 \&lng=en\&nrm=iso>. Acesso em: 19 mar. 2019. 


\section{PREVENÇÃO PARASITOSES INTENTINAIS E PEDICULOSE EM ESCOLARES: UM RELATO DE EXPERIÊNCIA}

Isabella Martinez Carvalho de Andrade ${ }^{1}$; Florence Mara Rosa ${ }^{2}$; Adaliza Furtado de Araújo ${ }^{3}$; Ana Paula Souza Oliveira ${ }^{4}$; Isabele Araújo Granato ${ }^{5}$; Letícia Silveira Meurer ${ }^{6}$.

${ }^{1}$ Graduação: Universidade Federal de Juiz de Fora/Faculdade de Medicina

${ }^{2}$ Graduação: Pontifícia Universidade Católica de Minas Gerais/Ciências Biológicas. Mestrado: Universidade

Federal de Minas Gerais/ Parasitologia. Doutorado: Universidade Federal de Minas Gerais / Parasitologia

${ }^{3}$ Graduação: Universidade Federal de Juiz de Fora/Faculdade de Medicina

${ }^{4}$ Graduação: Universidade Federal de Juiz de Fora/Faculdade de Medicina

${ }^{5}$ Graduação: Universidade Federal de Juiz de Fora/Faculdade de Medicina

${ }^{6}$ Graduação: Universidade Federal de Juiz de Fora/Faculdade de Medicina

Autor para correspondência: Isabella Martinez Carvalho de Andrade Email: isabella.andrade4@yahoo.com.br

\section{RESUMO}

Introdução: As parasitoses intestinais causadas por helmintos e a Pediculose são muito frequentes na infância, principalmente em pré-escolares e escolares de instituições públicas que residem em regiões onde o saneamento básico e as condições de higiene são precárias. Sendo assim, as medidas educativas quanto à higiene são fortes aliados no controle de tais parasitoses $^{1}$ e nesse sentido as atividades lúdicas são um ótimo recurso. Objetivos: Despertar o interesse das crianças pela prevenção das parasitoses intestinais e Pediculose. Relato de Experiência: O projeto de extensão "Prevenção de Parasitoses Intestinais e Pediculoses em Escolares" foi realizado na E. E. Santa Cândida e E. E. São Vicente de Paula, em Juiz de Fora, com 500 alunos regularmente matriculados nos $2^{\circ}, 3^{\circ}$ e $4^{\circ}$ anos do Ensino Fundamental. Inicialmente, foi ministrada uma palestra com slides e vídeos ${ }^{2}$ sobre as seguintes morbidades: Ascaridíase, Ancilostomíase, Tricuríase, Esquistossomose, Oxiuríase, e Pediculoses. Nesse momento, foi estabelecido um diálogo com os escolares e foram discutidos aspectos sobre a biologia do parasito, transmissão, sintomatologia e, principalmente a prevenção destas doenças. Após a palestra, os alunos eram divididos em grupos e o material lúdico confeccionado pelos extensionistas, como jogos de tabuleiro ${ }^{3}$, jogos de memória e caça palavras foram utilizados. Enquanto os jogos didáticos eram realizados, os escolares, revezadamente, foram levados a um laboratório para a visualização dos parasitos. Resultados e Discussão: Foi observado que o tema proposto despertou o interesse dos alunos, pois durante as palestras eram feitas várias perguntas. Nesse momento, algumas crianças faziam relatos próprios de determinadas parasitoses. A utilização de recursos de vídeo na palestra melhorou a aprendizagem e dinamizou a interação entre os escolares e o palestrante. Por meio das atividades lúdicas foi possível verificar o aprendizado dos alunos principalmente no que se refere às medidas de prevenção. A parte prática despertou grande interesse e excitação nos alunos, a grande maioria nunca tinha tido contato com o microscópio. Conclusão: $O$ relato de experiência mostra que é possível despertar o interesse dos alunos pela prevenção das parasitoses intestinais e pediculose, introduzindo-se hábitos de higiene e cuidados que contribuem para o controle da transmissão destas parasitoses ${ }^{4}$. Essa atividade de extensão também foi uma forma de integrar os acadêmicos da área de saúde com a população local.

Palavras-chave: parasitoses, pediculose, escolares. 


\section{Referências:}

1. DIAS, J. C. P. Problemas e Possibilidade de Participação Comunitária no Controle das Grandes Endemias no Brasil. CadSaúde publica 1998. p. 14(sup 2): 19-37

2. KRUSCHEWSKY, E. J. et al. Experiências pedagógicas de educação popular em saúde: a pedagogia tradicional versus a problematizadora. Saúde \&Amb. Rev. 2008 jul-dez. p. 4(2):160- 176.

3. SILVA, T. S., Leda, L. R. Intervenções educativas sobre parasitoses intestinais: aplicação de um jogo para alunos do ensino fundamental. Saúde \&Amb. Rev. 2012 juldez. p. 7(2):23-07.

4. VILA, A. C. D., Vila, V. S. C. Tendências da produção do conhecimento na educação em saúde no Brasil. Rev Latino-Am Enfermagem 2007. p. 15(6): 1177-83. 


\title{
RELATO DE CASO: SÍFILIS CONGÊNITA E SEPSE NEONATAL EM RECÉM-NASCIDO A TERMO
}

\author{
Luisa Couto Baptista ${ }^{1}$; Adaliza Furtado Araújo²; Isabele Araújo Granato ${ }^{3}$; Maria Eduarda \\ Luíza Pereira ${ }^{4}$; Miralva Aurora Galvão Carvalho ${ }^{5}$ Janaina Martins de Souza ${ }^{6}$. \\ 1 Graduação: Universidade Federal de Juiz de Fora/Faculdade de Medicina; \\ ${ }^{2}$ Graduação: Universidade Federal de Juiz de Fora/Faculdade de Medicina; \\ ${ }^{3}$ Graduação: Universidade Federal de Juiz de Fora/Faculdade de Medicina; \\ ${ }^{4}$ Graduação: Universidade Federal de Juiz de Fora/Faculdade de Medicina; \\ ${ }^{5}$ Graduação: Universidade Federal de Juiz de Fora/Faculdade de Medicina; \\ ${ }^{6}$ Graduação: Universidade federal de Juiz de Fora/Faculdade de Medicina.
}

Autor para correspondência: Luisa Couto Baptista

Email: lucoutob@hotmail.com

\section{RESUMO}

Introdução e Objetivos: A sífilis é uma IST de caráter sistêmico, curável, causada pelo Treponema pallidum, uma bactéria Gram-negativa do grupo das espiroquetas ${ }^{1}$. É transmitida por via sexual, vertical e raramente por transfusão sanguínea. A sífilis congênita (SC) corresponde à infecção do feto pelo Treponema pallidum, sendo transmitida por via transplacentária em qualquer momento da gestação ${ }^{2}$. A incidência dos casos de sífilis adquirida, sífilis gestacional e SC teve aumento de 32,7\%,20,9\% e 1,9\%, respectivamente, entre 2014 e $2015^{3}$. Para que uma gestante seja considerada adequadamente tratada, o tratamento deve ser completo, adequado ao estágio da doença, feito com penicilina e finalizado pelo menos 30 dias antes do parto, tendo sido o parceiro tratado concomitantemente ${ }^{4}$. No entanto, sabe-se que as dificuldades em tratar o parceiro são muitas, principalmente pela falta de acesso às dosagens mensais de VDRL e pela falta de adesão ao tratamento, constituindo um entrave para a prevenção da $\mathrm{SC}^{5}$. Descrever sinais e sintomas de um caso precoce de sepse neonatal associada a sífilis congênita em recém-nascido (RN) de gestante inadequadamente tratada. Material e Métodos: Análise retrospectiva de prontuário médico da gestante e relatório de alta hospitalar para coleta de dados, além de consulta médica em ambiente de Unidade Básica de Saúde consistindo em anamnese e exame físico completo do RN. Resultados e Discussão: FFP, 38 anos, feminino, tercigesta, obesa, histórico de pré-eclâmpsia na última gestação sendo necessário parto cesáreo de urgência há 10 anos. Realizou 7 consultas de pré-natal na atenção primária concomitantemente com acompanhamento em pré-natal de alto risco na atenção secundária. Detectada sorologia demonstrando VDRL positivo com titulação de 1:64 na $26^{\mathrm{a}}$ semana de gestação. Foi tratada com Penicilina G benzatina 2,4 milhões UI 1 ampola IM por semana, por 3 semanas (3 doses $=6$ doses de 1,2milhões), completando tratamento mais de 30 dias antes do parto. $\mathrm{O}$ parceiro realizou dosagem de VDRL negativa no início do acompanhamento, não sendo repetida a dosagem por falta de acesso e colaboração do mesmo, tornando a gestante inadequadamente tratada. Nascimento do RN a termo de 40 semanas, parto cesáreo, APGAR 6 e 9, peso ao nascimento 3620 gramas, comprimento: $52 \mathrm{~cm}$, perímetro cefálico de $36 \mathrm{~cm}$, perímetro torácico: $34 \mathrm{~cm}$ e perímetro abdominal: $35 \mathrm{~cm}$. Nasceu não vigoroso, hipotônico, em apneia, sendo necessária reanimação neonatal com Ventilação com Pressão Positiva. No parto, foi encontrada titulação de VDRL materno de 1:32. O RN possuía análise de LCR sem alterações, porém VDRL reagente no sangue periférico com titulação de 1:4, sendo prescrito Penicilina Cristalina IV associada à Amicacina IV por 10 dias. Permaneceu internado por 13 dias devido ao diagnóstico de sífilis congênita e sepse neonatal. Encontra-se atualmente em aleitamento materno, assintomático, em acompanhamento na unidade básica de saúde e com 
infectologia para detecção precoce de possíveis complicações. Conclusão: Concluímos que a crescente incidência de sífilis adquirida, gestacional e congênita no Brasil demonstra a complexidade do acompanhamento deste agravo. Diante das possíveis complicações ao feto, resultantes da SC, há necessidade de manejo estruturado e abordagem educacional efetiva das equipes de saúde a fim de assegurar a prevenção, bem como a adesão ao tratamento por parte dos usuários do sistema de saúde.

Palavras-chave: pré-natal, sífilis congênita, complicações.

\section{Referências:}

1- ANDRADE, A. L. M. B. et al. Late diagnosis of congenitaL syphiLis: a recurring reaLity in women and chiLdren heaLth care in BraziL. Revista Paulista de Pediatria, n. AHEAD, 2018. ISSN 0103-0582.

2- BRASIL. Ministério da Saúde. Secretaria de Vigilância em Saúde. Protocolo clínico e diretrizes terapêuticas para a prevenção da transmissão vertical de HIV, Sílifis e Hepatites virais. Brasília, DF, 2018.

3- GARBIN, A. J. Í. et al. Reemerging diseases in Brazil: sociodemographic and epidemiological characteristics of syphilis and its under-reporting. Revista da Sociedade Brasileira de Medicina Tropical, v. 52, 2019. ISSN 0037-8682. Disponível em: < http://www.scielo.br/scielo.php?script=sci_arttext\&pid=S0037$86822019000100615 \&$ nrm $=$ iso $>$.

4- BRASIL. Ministério da Saúde. Secretaria de Vigilância em Saúde. Diretrizes para o Controle de Súfilis Congênita. Brasília, DF, 2006.

5- ROCHA, A. F. B. et al. Management of sexual partners of pregnant women with syphilis in northeastern Brazil - a qualitative study. BMC Health Services Research, v. 19, n. 1, p. 65, January 24 2019. ISSN 1472-6963. Disponível em: < https://doi.org/10.1186/s12913-019$3910-\mathrm{y}>$. 


\title{
REVISÃO DE LITERATURA: IMPACTO DA AVALIAÇÃO DA ARTÉRIA CARÓTIDA NA ESTRATIFICAÇÃO DO RISCO CARDIOVASCULAR EM HIPERTENSOS
}

Gabriella Altomare Andrade ; Ana Luíza de Castro Carvalho²; Giulia Machado Caldeira Ardisson³ Luísa Vianna Cançado ${ }^{4}$; Ygor Felipe Leite de Souza ${ }^{5}$; Flávio Augusto Teixeira Ronzani ${ }^{6}$.

1 Acadêmica de medicina; Universidade Federal de Juiz de Fora;

2 Acadêmica de medicina; Universidade Federal de Juiz de Fora;

3 Acadêmica de medicina; Universidade Federal de Juiz de Fora;

${ }^{4}$ Acadêmica de medicina; Universidade Federal de Juiz de Fora;

${ }^{5}$ Acadêmico de medicina; Universidade Federal de Juiz de Fora;

${ }^{6}$ Graduação em medicina pela Universidade Federal de Juiz de Fora; especialista em Radiologia e Diagnóstico por imagem pela Associação Médica e pelo Colégio Brasileiro de Radiologia; mestrado em Saúde Brasileira pela Universidade Federal de Juiz de Fora.

\author{
Autor para correspondência: \\ Gabriella Altomare Andrade \\ E-mail: gabriella_altomare@hotmail.com
}

\section{RESUMO}

Introdução e Objetivos: Acometimentos metabólicos e cardiovasculares estão entre as principais causas de mortalidade mundial dos indivíduos adultos e idosos. A hipertensão arterial atinge 32,5\% dos indivíduos adultos e mais de $60 \%$ dos idosos, contribuindo direta ou indiretamente para $50 \%$ das mortes por doença cardiovascular ${ }^{1}$. O objetivo deste trabalho é analisar a efetividade da ultrassonografia da artéria carótida na estratificação do risco cardiovascular em pacientes hipertensos por meio de uma revisão literária. Material e Métodos: A análise de literatura se estabeleceu por meio de uma pesquisa nas bases de dados PubMed e LILACS pelas palavras chave: hipertensão, espessura íntimo-medial carotídea, estratificação de risco, ultrassom; no período de 12 de fevereiro 2019 a 15 de março de 2019. Foram excluídos artigos que não tratavam da temática central abordada na revisão: relação entre EIMC e o risco cardiovascular em pacientes hipertensos. Foi realizada uma síntese de resultados e de evidências, interpretando criticamente as pesquisas disponíveis sobre a medida ultrassonográfica para rastreio em hipertensos com alto risco cardiovascular. Resultados: Considerando ausência de dados brasileiros para uma avaliação mais precisa do risco cardiovascular na nossa população, deve-se evitar o uso de um único escore de risco para basear as decisões terapêuticas ${ }^{1}$. Embora a medida da espessura íntimo-medial carotídea (EIMC) seja considerada um marcador direto de aterosclerose subclínica, com importante valor potencial na estratificação de risco cardiovascular, ela não tem sido preconizada para uso rotineiro em indivíduos hipertensos ${ }^{2}$. Existe associação independente entre EIMC e incidência de doenças cerebrovascular e coronariana ${ }^{3}$, tornando a medida uma ferramenta valiosa na avaliação da aterosclerose e também do risco cardiovascular. Discussão: Nesse panorama a ultrassonografia carotídea surge como ferramenta diagnóstica, podendo ser utilizada com boas sensibilidade e especificidade, de acordo com o estudo ${ }^{4}$. A ecografia clínica permite boa visualização das camadas média e íntima da artéria carótida, um dos vasos mais afetados por alterações cardiovasculares. Com a ultrassonografia, espera-se maior sensibilidade na detecção de doenças cardiovasculares em comparação aos métodos de acompanhamento rotineiros já existentes. É válido ressaltar que a ecografia clínica é um exame de imagem relativamente barato, portátil, acessível, além de não apresentar contraindicações por possível prejuízo físico do paciente. Conclusão: O ultrassom de carótida que mede a EIMC é um exame com grande potencial para o rastreio de doenças 


\section{ANAIS DO XIII CONGRESSO MÉDICO ACADÊMICO, 2019; 08-61}

cardiovasculares podendo contribuir para um melhor prognóstico por detectá-las precocemente. Ademais, é um exame acessível, portátil e barato e por isso deve ser mais inserido na prática clínica diária.

Palavras-chave: Hipertensão. Espessura íntimo-medial carotídea. Estratificação de risco. Ultrassom.

\section{Referências:}

1- MALACHIAS, M.V.B. et al. $7^{a}$ Diretriz Brasileira de Hipertensão Arterial: Capítulo 3 Avaliação Clínica e Complementar. Arq. Bras. Cardiol., São Paulo, v. 107, n. 3, supl. 3, p. 2016. 14-17, set. Disponível em: $<$ http://www.scielo.br/scielo.php?script=sci_arttext\&pid=S0066782X2016004800014\&lng=pt\&nrm=iso>. Acesso em: 09 mar. 2019.

2- JÚNIOR, Francisco Das Chagas Monteiro et al. Prevalence of subclinical atherosclerosis and cardiovascular risk reclassification through measurement of carotid intima-media thickness in ambulatory hypertensive patients. Rev. Portuguesa de cardiologia, Portugual, v. 32, n. 12, p. 957-1054, dez. 2013. Disponível em: $<$ http://www.revportcardiol.org/pt-prevalencia-aterosclerose-subclinica-e-reclassificacaorisco-cardiovascular-pela-articulo-S2174204913002225 >. Acesso em: 1 nov. 2019.

3- TORRES, Felipe Soares et al. Medida da Espessura das Camadas íntima e Média das Artérias Carótidas para Avaliação do Risco Cardiovascular. Rev. Bras. Hipertensão, São Paulo, v.14, n.3, p. 167-171, jul. $2007 . \quad$ Disponível em: <http://departamentos.cardiol.br/dha/revista/14-3/09_medida.pdf>. Acesso em: 25 fev. 2019.

4- Wardlaw, J.M. et al. Accurate, practical and costeffective assessment of carotid stenosis in the UK. Health Technol Asses, v.10, n.30, p. 1-182, ago. 2006. Disponível em: <https://www.ncbi.nlm.nih.gov/pubmed/16904049>. Acesso em: 12 mar. 2019. 


\title{
EDUCAÇÃO CONTRA O TABACO(EAT) NA PREVENÇÃO DO TABAGISMO: UM RELATO DE EXPERIÊNCIA
}

\author{
Isabella Oliveira Lanzieri¹; Lorena Resende Oliveira²; Bárbara Bizzo Castelo ${ }^{3}$; Luísa Vianna \\ Cançado $^{4}$; Isabel Cristina Gonçalves Leite ${ }^{5}$ \\ ${ }^{1}$ Acadêmica de medicina; Universidade Federal de Juiz de Fora; \\ ${ }^{2}$ Acadêmica de medicina; Universidade Federal de Juiz de Fora; \\ ${ }^{3}$ Acadêmica de medicina; Universidade Federal de Juiz de Fora; \\ ${ }^{4}$ Acadêmica de medicina; Universidade Federal de Juiz de Fora; \\ ${ }^{5}$ Graduação em odontologia pela Universidade Federal de Juiz de Fora; mestrado em Saúde Pública pela Escola \\ Nacional de Saúde Pública; doutorado em Saúde Pública pela Escola Nacional de Saúde Pública.
}

Autor para correspondência: Isabella Oliveira Lanzieri

E-mail: isabellalanzieri1@gmail.com

\section{RESUMO}

Introdução: O EAT(Educação Contra o Tabaco) é um projeto movido por uma rede mundial conduzida por médicos e acadêmicos presente em mais de 70 faculdades, em 13 países, apoiado pela Escola de Medicina de Harvard e pelo Brigham and Women's Hospital. Atualmente, o tabagismo é a primeira causa de morte prevenível no mundo,e ressalta-se a importância da prevenção precoce do uso do tabaco, uma vez que essa atividade é considerada um dos maiores problemas de saúde coletiva do mundo, contribuindo para o surgimento de diversas patologias e internações gerando um custo expressivo para o SUS. Pesquisas recentes mostraram que a faixa etária provável de início do tabagismo, no Brasil, é de 12 a 17 anos, e , dessa forma, as intervenções do EAT são focadas nesses alunos. Objetivo: $\mathrm{O}$ objetivo do projeto encontra-se em atuar no combate ao tabagismo através de ação de prevenção na população jovem. Relato de Experiência: o EAT é um projeto de extensão da Universidade Federal de Juiz de Fora(UFJF) que usa metodologias baseadas em evidências, através da prevenção primária em estudantes por meio de aconselhamento, uso de softwares e materiais previamente testados e aplicados. O projeto é desenvolvido por meio de visitas dos acadêmicos às escolas da rede pública. As ações são divididas em etapas e são executadas em cerca de 1 hora, contando com iniciativas dinâmicas que usufruem do contato entre apresentador e aluno. Há mostra dos diversos tipos de consumo dos produtos de tabaco, artimanhas da indústria do tabaco, modelos comparativos de corpos saudáveis e debilitados pelo uso de tabaco, pôsteres sobre efeitos do cigarro na aparência física e o uso do aplicativo Smokerface (desenvolvido pelo EAT) que simula as modificações estéticas causadas por consumo de tabaco. Durante as ações,os alunos participantes são sempre convidados a participar ativamente das atividades propostas. Resultados e discussão: Em Juiz de Fora, estima-se que 150 alunos já participaram das ações do EAT, os quais se mostram interessados e receptivos. $O$ projeto tem enfoque nos benefícios de ser um não fumante, atuando de forma a estabelecer uma relação de confiança com os discentes, com uso de recursos atuais como o aplicativo $^{1}$ e convidando-os a conhecer cada instrumento e desvendando mitos. Há destaque para as implicações de curto prazo para os jovens como aspectos estéticos e financeiros, o que atrai o interesse dos alunos que se mostram sensibilizados com as apresentações.Pode-se destacar o relato um estudo científico em que tal abordagem na Alemanha² resultou em uma probabilidade seis vezes maior do jovem parar de fumar, sendo mais eficiente do que as focadas apenas na exposição de fatos prejudiciais do tabaco e de afecções de longo prazo como câncer e enfisema,as quais estão distantes da realidade da saúde do adolescente. No caso de Juiz de Fora, um acompanhamento contínuo dos alunos contemplados com as apresentações ainda se faz necessário para constatar a real eficiência do projeto na região. 


\section{ANAIS DO XIII CONGRESSO MÉDICO ACADÊMICO, 2019; 08-61}

Conclusão: Com isso, o projeto possibilita aos acadêmicos da UFJF a oportunidade de serem capacitados para a prevenção de forma correta ao tabagismo ao longo prazo,habilidade esta escassa nos currículos médicos ao longo do mundo, além de causarem impacto na comunidade através das ações dinâmicas de conscientização.

Palavras-chave: tabagismo; prevenção; juventude.

\section{Referências:}

1. FORMAGINI, Taynara Dutra Batista et al . A review of smartphone apps for smoking cessation available in Portuguese. Cad. Saúde Pública, Rio de Janeiro , v. 33, n. 2, e00178215, 2017 .

2. BRINKER, Titus $\mathbf{J}$ et al. Photoaging mobile apps: a novel opportunity for smoking cessation? J Med Internet Res, Giessen, v. 17,n. 7, 2015. 


\title{
CONHECIMENTOS DE USUÁRIAS DA ATENÇÃO BÁSICA DE JUIZ DE FORA SOBRE O RASTREAMENTO DAS NEOPLASIAS FEMININAS
}

\author{
Mikaela Santos Mascarenhas ${ }^{1}$; Davi da Costa Laurindo²; Lorena Pinholi de Morais²; Luan Viana Faria ${ }^{3}$; Mário \\ Círio Nogueira ${ }^{4}$. \\ ${ }^{1}$ Graduação; Faculdade de Medicina da Universidade Federal de Juiz de Fora; \\ ${ }^{2}$ Graduação; Faculdade de Medicina da Universidade Federal de Juiz de Fora; \\ ${ }^{3}$ Graduação; Faculdade de Odontologia da Universidade Federal de Juiz de Fora; \\ ${ }^{4}$ Docente da Faculdade de Medicina da Universidade Federal de Juiz de Fora.
}

\author{
Autor para correspondência: \\ Mikaela Santos Mascarenhas \\ Email: mikaelamascarenhas@gmail.com
}

\begin{abstract}
RESUMO
Introdução: Estima-se que, em 2018, o câncer de mama foi o mais incidente entre as mulheres brasileiras, representando $29,5 \%$ dos casos. Em terceiro lugar, está o câncer de colo de útero, com $8,1 \%$ das incidências ${ }^{1}$. Sabe-se que, quando diagnosticadas em estágios iniciais, ambas as neoplasias possuem alto potencial de cura. Dessa forma, destaca-se a importância de ações relacionadas ao controle do câncer feminino na atenção básica, por intermédio de estratégias na detecção precoce e rastreamento em vista ao cuidado integral à mulher. Objetivos: Investigar a adequação dos conhecimentos das usuárias das Unidades Básicas de Saúde (UBS) de Juiz de Fora sobre o rastreamento das neoplasias de colo de útero e de mama, tendo como referência as recomendações do Instituto Nacional de Câncer José Alencar Gomes da Silva (INCA), pertencente ao Ministério da Saúde, em suas diretrizes de $2015^{2}$ e $2016^{3}$, respectivamente. Material e métodos: Estudo descritivo, transversal, com questionário estruturado aplicado às usuárias das UBS na faixa etária de 25 a 69 anos. A amostra foi constituída de usuárias atendidas consecutivamente durante duas semanas na UBS Teixeiras, que contempla os bairros Bela Aurora, Cascatinha e Teixeiras. Foram estimadas prevalências de conhecimentos e de práticas adequadas. Resultados e discussão: Embora quase todas as mulheres já tenham ouvido falar destes exames, nenhuma apresentou conhecimento inteiramente adequado sobre o rastreamento destas neoplasias. Segundo as diretrizes mais recentes do INCA/Ministério da Saúde, a mulher deve iniciar o exame de rastreio do câncer de mama aos 50 anos de idade e realiza-lo bienalmente. Porém, apenas 5,7\% (IC95\%: 1,6\%9,8\%) das mulheres entrevistadas sabiam que a idade adequada de início do exame, enquanto a proporção de mulheres que acreditam que a mamografia deve começar a ser feita aos 40 anos foi de 45,2\% (IC95\%: 36,4\%-54,0\%). Além disso, a maioria das mulheres $(62,1 \%$; IC95\%: 53,5\%-70,7\%) acha que a periodicidade da mamografia deve ser anual, enquanto apenas 18,7\% (IC95\%: 11,8\%-25,6\%) conheciam a periodicidade adequada de 2 anos. Tal evidência pode estar relacionada a uma maior divulgação das recomendações da Sociedade Brasileira de Mastologia (SBM $)^{4}$ e da Federação Brasileira das Associações de Ginecologia e Obstetrícia (FEBRASGO) ${ }^{5}$, que diferem do posicionamento do INCA. Já em relação ao início e periodicidade do exame de rastreamento de câncer de colo de útero, as recomendações do INCA e FEBRASGO estão de acordo. A proporção de mulheres que sabiam que a idade recomendada para iniciar o rastreio com colpocitologia é aos 25 anos foi de 2,4\% (IC95\%: 0,0\%-5,2\%), enquanto as que acham que não há uma idade certa para iniciar a colpocitologia de rastreamento foi de 54,0\% (IC95\%: 45,2\%-62,8\%). Esta última resposta teve associação com o grau de escolaridade das mulheres, com as de ensino médio completo ou superior em maior proporção $(67,2 \%$; IC95\%: 55,1\%-79.4\%) e as de ensino fundamental completo com a menor proporção $(28,6 \%$; IC95\%: 9,2\%-48,0\%). Por fim, a maioria das mulheres $(70,2 \%$; IC95\%: $62,1 \%-78,2 \%$ ) acreditam que a colpocitologia deve ser feita anualmente e nenhuma
\end{abstract}


acertou a periodicidade correta de 3 anos. Conclusão: O conhecimento das usuárias da atenção básica ainda é muito precário sobre o rastreamento das neoplasias femininas. Ações de educação em saúde podem reverter esta situação e potencialmente levar a um impacto positivo na saúde destas populações.

Palavras-chave: neoplasia; mulher; atenção primária.

Referências:

1- INSTITUTO NACIONAL DE CÂNCER JOSÉ ALENCAR GOMES DA SILVA. Estimativa 2018: incidência de câncer no Brasil. Rio de Janeiro: INCA, 2017.

2- INSTITUTO NACIONAL DE CÂNCER JOSÉ ALENCAR GOMES DA SILVA. Diretrizes para a detecção precoce do câncer de mama no Brasil. Rio de Janeiro: INCA, 2015.

3- INSTITUTO NACIONAL DE CÂNCER JOSÉ ALENCAR GOMES DA SILVA. Diretrizes brasileiras para o rastreamento do câncer do colo do útero - 2. ed. rev. atual. Rio de Janeiro: INCA, 2016.

4- VIEIRA, Sabas Carlos. Câncer de mama: Consenso da Sociedade Brasileira de Mastologia - Regional Piauí - 2017. Teresina: EDUFPI, 2017.

5- FEDERAÇÃO BRASILEIRA DAS ASSOCIAÇÕES DE GINECOLOGIA E OBSTETRÍCIA. Rastreio, diagnóstico e tratamento do câncer de colo de útero. Série Orientações e Recomendações FEBRASGO. v. 1, n. 2, jan. 2016. São Paulo, 2017. 


\title{
RELATO DE CASO: DOENÇA CELÍACA EM ADULTOS
}

\author{
Yara Mônica da Silva Oliveira苂 Luisa Couto Baptista²; Maria Eduarda Luíza Pereira²; \\ Mariana Faceroli Oliveira²; Patrik Míkelos de Castro Lanes²; Vitor Rocha Couto ${ }^{3}$. \\ ${ }^{1}$ Graduação; Faculdade de Medicina da Universidade Federal de Juiz de Fora; \\ ${ }^{2}$ Graduação; Faculdade de Medicina da Universidade Federal de Juiz de Fora; \\ ${ }^{3}$ Docente da Faculdade de Medicina da Universidade Federal de Juiz de Fora.
}

Autor para correspondência:

Yara Mônica da Silva Oliveira

Email: yara.monica@hotmail.com

\section{RESUMO}

Introdução e Objetivos: A doença celíaca é uma enteropatia crônica, prevalente no sexo feminino, que ocorre por resposta imunológica inapropriada dos linfócitos $\mathrm{T}$ ao glúten contido na farinha de trigo e outros cereais. Afeta primariamente o intestino delgado em pacientes com predisposição genética. Observa-se aumento da prevalência da doença nos últimos 50 anos do diagnóstico nos últimos 10 anos. O quadro clínico é variado, podendo ir desde manifestações gastrointestinais até sintomas extraintestinais. Alguns pacientes, inclusive, podem ser assintomáticos, manifestando sintomas atípicos ou a forma silenciosa da doença. Seu diagnóstico ainda é um desafio para muitos serviços médicos, e pode ter sua investigação inicial através da dosagem de anticorpos específicos no soro, como anticorpos antiendomísio (EMA) e antitransglutaminase tecidual (anti-tTG). Apesar do papel das sorologias no diagnóstico, a sua confirmação se dá por meio de biópsia da mucosa duodenal, que pode apresentar como achados sugestivos infiltrado local associado a atrofia vilosa mucosa total ou parcial. Ambos os testes devem ser realizados com o paciente em dieta com glúten. $\mathrm{O}$ tratamento é feito pela retirada total do glúten da dieta. Novas terapias têm sido estudadas, mas ainda não foram comprovadas para uso em pacientes. O objetivo do presente trabalho é relatar e estudar o caso de uma paciente portadora de Doença Celíaca com diagnóstico tardio, visto que é uma doença subdiagnosticada e pouco conhecida, inclusive pela própria população médica. Material e Métodos: As informações foram obtidas por meio de revisão de prontuário e revisão de literatura. Resultados e Discussão: C.F.C, feminino, 36 anos, iniciou quadro de queda capilar intensa associada a fraqueza, astenia e câimbras em MMII. Apresentava história pregressa de sintomas gastrointestinais inespecíficos e esporádicos há 6 anos. Realizou exames laboratoriais, incluindo hemograma, que revelou anemia ferropriva severa. Após investigação médica, foi detectado anticorpo específico para doença celíaca e o diagnóstico confirmado por biópsia de mucosa duodenal através de endoscopia digestiva alta (EDA). Paciente iniciou dieta livre de glúten, com melhora dos sintomas. A clínica presente na paciente pode ser justificada pelo quadro inflamatório intestinal que resulta na síndrome de má absorção com deficiência de ferro, vitaminas e íons. A avaliação diagnóstica feita segue os padrões recomendados de realização de biópsia frente ao anticorpo positivo. As manifestações inespecíficas e espaçadas podem ter contribuído para o retardo do diagnóstico, levando ao desconforto prolongado da paciente e o risco de desenvolvimento de complicações, como neoplasias. Conclusão: Atualmente, há aumento da prevalência da doença celíaca mundialmente, com destaque para o crescimento das formas atípicas da afecção, o que explica o diagnóstico tardio. O tratamento efetivo é a restrição total ao glúten da dieta, sendo capaz de gerar melhora sintomática e prevenção de complicações da doença. Por ser uma doença relacionada a outras patologias, como infertilidade, osteoporose, tireoidites, anemias carenciais, manifestações psiquiátricas, neurológicas, e maior risco de desenvolvimento de neoplasias, o diagnóstico precoce, a implementação da terapêutica e o seguimento da patologia devem ser abordados, melhorando a qualidade de vida e o prognóstico dos 


\section{ANAIS DO XIII CONGRESSO MÉDICO ACADÊMICO, 2019; 08-61}

portadores de doença celíaca.

Palavras chave: doença celíaca, manifestação atípica, diagnóstico tardio.

\section{Referências:}

1. RUBIO-TAPIA, Alberto et al. ACG clinical guidelines: diagnosis and management of celiac disease. The American Journal of Gastroenterology, v. 108, n. 5, p. 656, 2013.

2. PEDRO, N. et al. Doença celíaca: revisão de conceitos e novos desenvolvimentos. 2009.

3. LIU, Shinfay Maximilian et al. Doença celíaca. Rev Med Minas Gerais, v. 24, n. Supl2, p. S38-S45, 2014.

4. GAMA E SILVA, Tatiana Sudbrack; FURLANETTO, Tania Weber. Diagnóstico de Doença Celíaca em Adultos. Revista da Associação Médica Brasileira, [S. l.], 2010. 


\section{AMBULATÓRIO DE ATENÇÃO A CRIANÇAS E ADOLESCENTES COM TRANSTORNO DO ESPECTRO AUTISTA: RELATO DE EXPERIÊNCIA}

Ana Teresa Dias Albino Destro de Macêdo ${ }^{1}$; Gabriela Ribeiro G. Bolina Batista ${ }^{1}$; Ângela Caroline Dias Albino Destro de Macêdo'; Márcia Helena Fávero de Souza

${ }^{1}$ Discentes do curso de medicina da UFJF

2 Docente da Faculdade de Medicina da UFJF.

Autor para correspondência:

Ana Teresa Dias Albino Destro de Macêdo

E-mail: anateresadiasmacedo@gmail.com

\section{RESUMO}

Introdução: O Transtorno do Espectro do Autismo (TEA) engloba um grupo de transtornos do desenvolvimento, cujas características nucleares envolvem déficits na interação social, na comunicação e no comportamento, em níveis de gravidade variados entre os portadores. Estudos têm comprovado que sintomas de TEA, geralmente, são passíveis de identificação antes dos 12 meses de idade, tornando-se mais estáveis entre os 18 e 24 meses. A identificação dos sinais precoces do autismo é crucial para um diagnóstico e, consequentemente, para uma intervenção precoce. O que vai significar melhores perspectivas prognósticas. Objetivos: Trata-se de um relato de experiência de um projeto de extensão desenvolvido no Hospital Universitário de Juiz de Fora, que teve como um dos objetivos criar um ambiente de orientação e divulgação sobre os sinais precoces do TEA. Relato de Experiência: Foram realizadas apresentações em ambiente de sala de espera para os usuários do sistema. Após uma revisão bibliográfica sobre o tema, foram criadas três linhas de desenvolvimento infantil: a linha da interação social, da linguagem e do brincar e um folder explicativo para divulgação do tema. Em um primeiro momento foram expostos e explicados os marcos do desenvolvimento esperados dentro daquela linha, para cada faixa etária, chamando a atenção para possíveis alterações encontradas em crianças com TEA. Em seguida, era aberto um momento para dúvidas, compartilhamento de ideias e relatos por parte do público. A apresentação atingia em média 40 pessoas, incluindo diferentes faixas etárias, pacientes de outras especialidades, acompanhantes e profissionais do Hospital Universitário. Percebemos que o tema despertava interesse e dúvidas. Os principais questionamentos foram as características do transtorno (principalmente no que diz respeito a seu diagnóstico e tratamento), como identificar sinais precoces e os profissionais de saúde envolvidos no fluxo do sistema para atendimento destes pacientes. Houve relatos espontâneos de mães de pacientes com TEA, sobre quais foram os marcos percebidos na criança e sua evolução durante o tratamento. Resultados e Discussão: Percebemos o pouco domínio da população quanto aos marcos esperados do desenvolvimento das crianças para cada faixa etária e que há grande dificuldade de percepção dos sinais e sintomas na faixa etária de início das alterações do TEA (entre 12 e 24 meses). Observamos, assim como demonstram os estudos, que o atraso no desenvolvimento da comunicação e linguagem é o sintoma mais relatado pelos pais. Todavia, embora menos reconhecidos, comprometimentos sociais são mais precoces e melhores indicadores para o diagnóstico. Ainda, percebemos que muitas vezes as dúvidas são tiradas com a população leiga, comparando o desenvolvimento de outras crianças, o que, muitas vezes, atrasa o diagnóstico e o tratamento destes pacientes. Conclusão: Acreditamos ser de grande importância a elaboração de trabalhos que busquem divulgar o tema e orientar a população, de modo a aumentar a identificação dos sinais precoces do TEA. Cremos que intervenções devam ser feitas também a nível educacional e de saúde, para capacitação de 
profissionais da educação e da rede básica de saúde, para melhor identificação dos sinais precoces do TEA, por serem esses os mais próximos das crianças e dos seus familiares.

Palavras-chave: Transtorno do Espectro do Autismo; Diagnóstico; Tratamento.

\section{Referências:}

1. SECRETARIA De SAÚde do ESTADO DE SÃO PAUlO. Protocolo do Estado de São Paulo de Diagnóstico Tratamento e Encaminhamento de Pacientes com Transtorno do Espectro Autista (TEA). 1a Edição. Editora: SEDPcD. 2013, São Paulo.

2. MINISTÉRIO DA SAÚDE, Secretaria de Atenção à Saúde Departamento de Ações Programáticas Estratégicas. Diretrizes de Atenção à Reabilitação da Pessoa com Transtornos do Espectro do Autismo (TEA). $1^{\text {a }}$ edição. Brasília - DF, 2014. 


\title{
PROJETO DE EXTENSÃO “SAÚDE E ESPIRITUALIDADE”
}

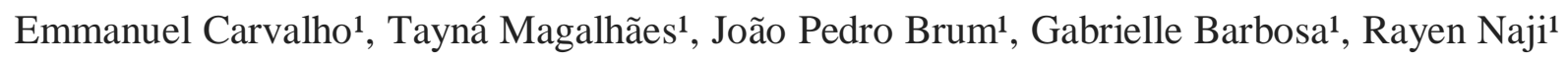 \\ e Marcelo Maroco Cruzeiro ${ }^{2}$ \\ ${ }_{1}^{1}$ Discentes do curso de medicina da UFJF \\ ${ }^{2}$ Professor Associado da UFJF, Pós-doutorando do NUPES
}

\author{
Autor para correspondência: \\ Emmanuel Carvalho \\ E-mail: emmanucarvalho@gmail.com
}

\section{RESUMO}

Introdução: A contemporaneidade traz à luz novos desafios para os profissionais de saúde inter-relações entre saúde e espiritualidade. Para além dos limites da fisiologia corporal, considerar o ser humano integral representa um aspecto importante da formação profissional. ${ }^{1}$ A cura e a prevenção de doenças sempre estiveram ligadas a práticas religiosas, mas com o advento da medicina científica, esses aspectos foram desvinculados. Os profissionais de saúde passaram a ser formados pelo paradigma científico da modernidade, separando corpo e mente. ${ }^{2}$ A religião não desapareceu. É necessário que os profissionais de saúde recuperem a dimensão devida do aspecto da religiosidade/espiritualidade no âmbito da saúde e da doença. Objetivos: Propor debates com a comunidade sobre temas de importância na área de saúde e espiritualidade, dando voz para experiências individuais. Integrar o assunto no meio da graduação e pós-graduação da UFJF e desenvolver a compreensão de que a dimensão espiritual tem relação com o processo saúde-doença, além de ser uma forma terapêutica. Relato de experiência: O projeto, iniciado em setembro de 2018, se baseia em "Rodas de Conversa" onde a comunidade é convidada a participar da discussão de modo ativo, após a exposição de um palestrante. Os membros do projeto fazem reuniões quinzenais para decidir os temas das rodas de conversa, bem como definir datas e palestrantes. A divulgação é feita por redes sociais e banners espalhados em diferentes pontos da UFJF. As inscrições para participar da roda são feitas através de formulário eletrônico. Com o objetivo de tornar o conteúdo das rodas de conversa público, são transmitidas ao vivo via Facebook e depois disponibilizadas na íntegra no canal do YouTube. Resultados e discussão: O projeto tenta trazer para o meio acadêmico da UFJF um dos elementos que tem sido colocado como fundamental no tratamento dos doentes, a espiritualidade, que juntamente com as crenças culturais e práticas se mostram importantes para a saúde e bem estar de muitos pacientes. Através das nossas rodas de conversa, podemos trocar experiências com profissionais e o público, buscando unir o aprendizado de ambos os lados. Os acadêmicos têm um contato direto com as experiências pessoais da comunidade, sendo possível a inserção desse conhecimento na visão do ser biopsicossocial. A atividade se mostra satisfatória quanto a criação de um grupo de reflexão permanente para o desenvolvimento de uma mentalidade que vise a inserção da espiritualidade como elemento em uma assistência mais humanizada na saúde. Já aconteceram três encontros, sendo que a primeira roda de conversa tratou da "Associação entre religiosidade/espiritualidade no enfrentamento da doença" - tratado pela psicóloga Janaína Siqueira. Dezesseis pessoas participaram. A segunda roda de conversa "Experiência de Quase Morte e seu impacto" - foi coordenada pelos professores Monalisa Silva e Marcelo Maroco Cruzeiro - com 52 participantes. Praticamente todos que compareceram a primeira roda de conversa, compareceram na segunda. A terceira roda de conversa - "Morte e morrer: um espaço para reflexão" - foi conduzida pelos alunos Emmanuel Carvalho, João Pedro Brum e Tayná Magalhães, e contou com 18 participantes. Conclusão: Apesar de recente, o projeto se mostra fortalecido ao servir à reflexão sobre os 
temas junto com comunidade, com participação crescente e dialogando sobre outro aspecto da dimensão humana, atualmente alijado das atividades acadêmicas.

Palavras-chave: saúde, espiritualidade, comunidade.

\section{Referências:}

1. PUCHALSKI, Christina M. The role of spirituality in health care. In: Baylor University Medical Center Proceedings. Taylor \& Francis, 2001. p. 352-357.

2. NUMBERS, Ronald L. Mitos e verdades em ciência e religião: uma perspectiva histórica. Archives of Clinical Psychiatry, v. 36, n. 6, p. 250-255, 2009. 


\title{
RELATO DE CASO: LINFOMA NÃO HODGKIN DE CÉLULAS DO MANTO
}

\author{
Leda Caldeira de Souza1; Lorena Pinholi de Moraisi' ${ }^{1}$ Gabrielle da Silva Barbosa ${ }^{1}$; Kátia \\ Regina Alves Lopes² \\ ${ }^{1}$ Acadêmicos de Medicina da Universidade Federal de Juiz de Fora (UFJF) \\ ${ }^{2}$ Professora da Faculdade de Medicina da Universidade Federal de Juiz de Fora (UFJF)
}

Autor para correspondência:

Leda Caldeira de Souza

E-mail: ledacaldeiradesouza@gmail.com

\section{RESUMO}

Intodução: Os linfomas não Hodgkin (LNH) constituem um grupo muito heterogênio de neoplasias do sistema linforeticular. O Linfoma de Células do Manto (LMC) é uma doença linfoproliferativa de células B maduras de caráter agressivo que corresponde a aproximadamente $6 \%$ de todos os $\mathrm{LNH}$. Trata-se de uma neoplasia com pequena chance de cura, com terapias atuais insatisfatórias, apresentando $25 \%$ de sobrevida média em 5 anos. $70 \%$ dos casos são diagnosticados em estágio IV de Ann Arbor. Objetivos: relatar um caso de Linfoma não Hodgkin de Células do Manto diagnosticado em estágio IV-B e salientar a importância de seu conhecimento, analisando as características clínicas e imunofenotípicas. Material e Métodos: Atendimento em enfermaria e revisão de prontuário médico. Resultados: J.N.S, 60 anos, sexo feminino, branca, com história de hematêmese de melhora espontânea, que se repetiu após 1 ano, associada a melena, odinofagia, disfonia e perda ponderal de $20 \mathrm{~kg}$. No exame físico foi evidenciado hipertrofia de Anel de Waldeyer (com risco de obstrução de via aérea superior), síndrome consuptiva, linfonodomegalia em cadeias cervical posterior, supraclavicular, axilar e inguinal bilateralmente e submandibular. Associado identificou-se síndrome dispéptica com sinais de alarme (emagrecimento, idade e anemia). Exames laboratoriais evidenciaram anemia (hemoglobina 9,0 g/dl). $\mathrm{Na}$ endoscopia digestiva alta foram visualizadas varizes esofágicas, gastrite endoscópica erosiva plana moderada e pólipos duodenais. Colonoscopia com lesões de reto. Exames de imagem evidenciaram linfonodomegalias em região cervical, de hilo pulmonar, em região ilíacoinguinal, em hilo hepático e intercavoaórticos, ascite moderada, sinais de hepatopatia crônica e hipertensão portal. O diagnóstico foi realizado com biópsia das lesões de reto e bulbo duodenal que apresentaram infiltração em padrão nodular em crescimento, por células linfoides de núcleos convolutos angulados e citoplasma escasso. A expressão de CD20, CD5 e cliclina D1 na imunohistoquímica são consistentes com Linfoma de células do Manto.A biópsia de medula óssea mostrou infiltração por linfoma .Foi iniciada quimioterapia com esquema CHOP (ciclofosfamida, adriamicina, vincristina e prednisona). Discussão: $O$ linfoma de células do manto está entre as neoplasias linfoides de células B maduras, a qual se apresenta com maior frequência nos estágios III-IV de Ann Arbor. Os pacientes geralmente têm média de idade de 60 anos, apresentando linfonodomegalia generalizada, hepatoesplenomegalia, sintomas B (febre, sudorese noturna e perda ponderal superior a $10 \%$ nos últimos 6 meses) e acometimento da medula óssea, com possibilidade de acometimento do anel de Waldeyer e do trato gastrointestinal. No caso relatado, a paciente apresentava um quadro clínico característico associado a sintomas gastrointestinais, além de sintomas B (perda ponderal), em estágio IV-B. O tratamento foi compatível com o regime mais utilizado (CHOP). Conclusão: A paciente apresentou um quadro de linfoma de células do manto com sintomatologia gastrointestinal. Trata-se do linfoma não Hodgkin que apresenta um dos piores prognósticos, com sobrevida que geralmente não ultrapassa 5 anos, o que evidencia a necessidade de seu conhecimento, para o diagnóstico mais precoce possível. 
Palavras-chave: Linfoma não-Hodgkin; células do manto.

\section{Referências:}

1- HOFFBRAND, A. V.; MOSS, P. A. H.Fundamentos em Hematologia. Traduzido e revisado por Renato Failace. 6. ed.Porto Alegre: Artmed, 2013.

2- KI, Maron El. Linfoma Não-Hodking de Células do Manto: Relato de Caso. Revista Científica da Faculdade de Medicina de Campos, v. 5. 2010. Disponível em < http://www.fmc.br/revista/V5N1P02-09.pdf>. acessos em 24 mar. 2019.

3- BORGES, Elisabete Pinto; PINHEIRO, Maria João; MOREIRA, Daniela. Um caso clínico de linfoma de células do manto. Rev Port Med Geral Fam, Lisboa, v. 31, n. 4, p. 267-270, ago. 2015 . Disponível em $<\mathrm{http}: / / \mathrm{www}$.scielo.mec.pt/scielo.php?script=sci_arttext\&pid=S2182$51732015000400006 \& \operatorname{lng}=$ pt\&nrm=iso $>$. acessos em 24 mar. 2019.

4- CAMPOS, Luciano Carvalho; ANDRADE, Diocésio Alves Pinto de. Linfoma nãoHodgkin de células do manto: relato de caso. Revista Médica de Minas Gerais, Belo Horizonte, v. 19, p.177-179, nov. 2009. 


\title{
RELATO DE CASO: SÍNDROME DE TAKOTSUBO, A CARDIOMIOPATIA EMERGENTE DA ERA MODERNA
}

\author{
Tayná Moreira ${ }^{1}$; Tarlline Ribeiro Sirilo; Guilherme Neif Vieira Musse² \\ ${ }^{1}$ Acadêmica de medicina; Universidade Federal de Juiz de Fora; \\ ${ }^{2}$ Acadêmica de medicina; Universidade Federal de Juiz de Fora; \\ ${ }^{3}$ Médico cardiologista residente de cardiologia do esporte no Hospital Sírio Libanês
}

Autor para correspondência:

Tayná Moreira

E-mail: tayna-moreira@hotmail.com

\section{RESUMO}

Introdução e objetivos: A síndrome de Takotsubo (ou síndrome do coração partido) é uma causa rara, porém cada vez mais reconhecida de insuficiência cardíaca aguda ${ }^{1,2}$, desencadeada $\left(85 \%\right.$ dos $\left.\operatorname{casos}^{3}\right)$ por situações de estresse, emocional ou físico, nas quais apresentam uma liberação de catecolaminas que atuam diretamente na parede do miocárdio e desencadeiam uma disfunção ventricular esquerda transitória. Tem maior incidência em mulheres pós-menopausa ${ }^{3}$ e sua apresentação é semelhante à síndrome coronariana aguda (SCA). Contudo, há ausência de lesões significativas ${ }^{4}$ na cineangiocoronariografia. Dado a necessidade de compreender qual o real impacto dos estímulos psicológicos e a possível natureza multifatorial dessa doença ${ }^{5}$, a finalidade desse trabalho é chamar atenção para o caso de uma paciente que evoluiu com tal desfecho após intenso estresse emocional. Relato de experiência: Mulher, 59 anos, previamente hipertensa, admitida com quadro súbito de dor retroesternal, sudorese profusa e sensação de mal-estar. Relatou que está em processo de habilitação para direção e ao retirar o carro da garagem passou por intenso estresse que coincidiu com o início do quadro. Na emergência, evidenciou-se: ECG com supradesnivelamento do segmento ST em parede anterior e marcadores de necrose miocárdica com elevação de Troponina I $(10,0$ ng/ml, VR<1,2) e CKMB massa $(16,0$ ng/ml, VR<6). Diante do quadro típico de infarto agudo do miocárdio, foi indicada cineangiocoronariografia de urgência. Durante a ventriculografia, concluiu-se que o VE apresentava volume sistólico e diastólico final aumentado devido a grande aneurisma de toda a parede ântero-apical, com balonamento desse segmento cardíaco durante a sístole ventricular. As discretas lesões coronarianas observadas não eram compatíveis com irrigação da parede anterior. Ecodopplercardiograma realizado após internação em CTI revelou disfunção sistólica moderada do VE à custa de hipocinesia importante da porção apical de todas as paredes. Assim, tais achados corroboraram para o diagnóstico da síndrome de Takotsubo. A paciente teve evolução favorável e recuperou completamente a função ventricular. Discussão: Os critérios diagnósticos ${ }^{4}$ desta forma reversível de insuficiência ventricular esquerda ainda estão em evolução. Os mais aceitos atualmente consistem em: evidência de hipocinesia transitória, acinesia ou discinesia nos segmentos médios e/ou apical do ventrículo esquerdo por meio de ventriculografia e/ou ecocardiograma, ausência de doença coronariana obstrutiva, novas anormalidades no ECG e ausência de feocromocitoma e miocardite. O caso apresentado preencheu todos os critérios. O prognóstico é bom, com recuperação morfofuncional do miocárdio em até um mês $\mathrm{s}^{3,4}$. Contudo, apesar da fisiopatologia ainda não estar totalmente elucidada, pesquisas associam tal condição a traços como inibição social, estresse psicológico crônico e transtornos ansioso-depressivos ${ }^{5,6}$. Ou seja, o número crescente de distúrbios psicossomáticos evidenciados na população fazem associação com o aumento da incidência da síndrome. Conclusão: Em contexto atual, a síndrome de Takotsubo deve ser considerada como um notável diagnóstico diferencial na abordagem da dor torácica aguda. O profissional deve se 


\section{ANAIS DO XIII CONGRESSO MÉDICO ACADÊMICO, 2019; 08-61}

manter alerta para tal possibilidade diagnóstica, especialmente em mulheres na pósmenopausa na vigência de alguma situação de estresse sistêmico, que se apresentem inicialmente com quadro clínico de SCA.

Palavras-chave: Cardiomiopatia de Takotsubo, estresse psicológico, dor no peito, contração miocárdica.

\section{Referências:}

1. Y-Hassan S, Tornvall P. Epidemiology, pathogenesis, and management of takotsubo syndrome. Clin Auton Res. 2017;28(1):53-65.

2. Santoro F, Ieva R, Ferraretti A, Ienco V, Carpagnano G, Lodispoto M, et al. Safety and feasibility of levosimendan administration in takotsubo cardiomyopathy: a case series. Cardiovasc Ther. 2013;31:e133-e137. doi: 10.1111/1755-5922.12047.

3. Akashi YJ, Nef HM, Lyon AR. Epidemiology and pathophysiology of Takotsubo syndrome. Nat Rev Cardiol. 2015;12:387-97

4. Madhavan M, Prasad A. Proposed Mayo Clinic criteria for the diagnosis of Tako-Tsubo cardiomyopathy and long-term prognosis. Herz. 2010;35:240-3.

5. Delmas C, Lairez O, Mulin E, et al. Anxiodepressive disorders and chronic psychological stress are associated with Tako-Tsubo cardiomyopathy- New Physiopathological Hypothesis. Circ J. 2013;77:175-80.

6. Kastaun S, Schwarz NP, Juenemann M, et al. Cortisol awakening and stress response, personality and psychiatric profiles in patients with takotsubo cardiomyopathy. Heart. 2014;100:1786-92 


\title{
RELATO DE CASO: LESÃO MULTILIGAMENTAR DO JOELHO COM TRATAMENTO POR ENXERTO AUTÓLOGO COM RETORNO AO ESPORTE
}

\author{
Heitor Teixeira Alves Carvalho ${ }^{\mathbf{1}}$; Petrus Ferreira Renó ${ }^{1}$; Luís Fernando Diniz do Carmo ${ }^{1}$; \\ Cláudio Otávio da Silva Bernardes 2; Samuel Lopes Mendes ${ }^{3}$. \\ 1 Acadêmica de medicina; Universidade Federal de Juiz de Fora; \\ ${ }^{2}$ Hospital Monte Sinai, Juiz de Fora, MG, Brasil; \\ ${ }^{3}$ Santa Casa de Misericórdia de Juiz de Fora, Juiz de Fora, MG, Brasil
}

Autor para correspondência:

Heitor Teixeira Alves Carvalho

E-mail: heitorcarvalhoufjf@gmail.com

\section{RESUMO}

Introdução: A Lesão Multiligamentar do Joelho (LMJ) é um condição de rara afecção, representando $0,02 \%$ de todas lesões ortopédicas. Traz grande déficit funcional e de morbidade devido ao seu dano articular, neurológico e vascular. ${ }^{1}$ Junto com a Lesão do Ligamento Cruzado Posterior (LCP), do Ligamento Cruzado Anterior (LCA) e de um Ligamento Colateral, mais prevalente o Medial, há o estabelecimento da LMJ (classificadas em Schenck KDIII - LCP-LCA-Ligamento Colateral). ${ }^{2}$ O quadro advém de traumas (90\%), majoritariamente acidentes automobilístico, e com maior prevalência em homens jovens $(75 \%){ }^{3}$ Os resultados após LMJ possuem pobre descrição na literatura devido sua rara ocorrência. Contudo, técnicas em Fixação Tibial "tibial inlay technique", e do Túnel Transtibial "trans-tibial tunnel", o uso de banda simples ou banda dupla, de enxertos autólogos e homólogos e quais utilizar, bem como o tempo "timing" cirúrgico são variáveis que possuem bastante controvérsias e discussão na literatura. Entretanto, o tratamento cirúrgico, atualmente, possui notáveis resultados diante o tratamento conservador. ${ }^{4}$ Em todos os estudos analisados houve grande significância na avaliação Lysholm após a cirurgia, apresentando melhora funcional e de estabilidade no joelho, bem como retorno ao esporte. Objetivos: Apresentar um relato de caso de intervenção cirúrgica precoce de paciente com LMJ com avaliação funcional dos resultados, obtidos através do Questionário de Lysholm, e com reabilitação de paciente até o retorno ao esporte. Material e métodos: Trata-se de relato de um caso de tratamento cirúrgico de lesão multiligamentar (LCA, LCP e ligamento colateral medial) traumática do joelho em paciente masculino, estudante, 18 anos e morador de cidade do interior de MG. A abordagem cirúrgica foi realizada em tempo único, com utilização de enxertos autólogos, optando por tendões flexores contralaterais para o ligamento cruzado posterior, tendão patelar para o ligamento cruzado anterior e o semitendíneo sem desinserção da tíbia para o ligamento colateral medial. Este é um trabalho descritivo da abordagem cirúrgica utilizada no tratamento definitivo de lesão traumática multiligamentar de joelho com análise quantitativa e qualitativa dos dados. As informações foram obtidas por meio de análise do prontuário do paciente, entrevista com o mesmo, aplicação do Questionário de Lysholm e revisão da literatura. Resultados e discussão: Paciente evoluiu com pós-operatório imediato satisfatório e sem intercorrências. Relatou leve dor na região da cirurgia durante a primeira semana. Foi instruído um período de carga protegida durante 2 meses e após este iniciou-se o tratamento fisioterápico, realizado 2 vezes por semana durante 8 meses. Com 1 ano e 5 meses de pós-operatório, paciente relata que consegue fazer todas suas atividades usuais e práticas esportivas sem dificuldades, tais como andar de bicicleta e jogar futebol. Apresenta uma pontuação de 95 pontos no Questionário de Lysholm, em comparação com uma pontuação de 23 pontos anterior à cirurgia. Conclusão: A opção pela abordagem em tempo cirúrgico único e utilização de enxertos autólogos é ótima opção para o tratamento das LMJ, especialmente 
em nosso meio, onde dispomos de pouco acesso ao aloenxerto. Os resultados observados foram significativos e corroboram essa conclusão, com obtenção de pontuação classificada como excelente de acordo com o Questionário de Lysholm e retorno pleno ao esporte.

Palavras-chave: lesão multiligamentar; enxerto autólogo; retorno ao esporte.

\section{Referências:}

1 - GOMES, R. J. Dissertação - Case Report Mestrado Integrado em Medicina LESÃO MULTILIGAMENTAR DO JOELHO : RELATO DE CASO E REVISÃO DA LITERATURA Porto 2017. 2017.

2 - RICHTER, D.; WASCHER, D. C.; SCHENCK, R. C. A novel posteromedial approach for tibial inlay PCL reconstruction in KDIIIM injuries: Avoiding prone patient positioning. Clinical Orthopaedics and Related Research, v. 472, n. 9, p. 2680 - 2690, 2014. ISSN 15281132.

3 - VAQUERO-PICADO, A.; RODRIGUEZ-MERCHÁN, E. C. Isolated posterior cruciate ligament tears: an update of management. EFORT Open Reviews, v. 2, n. 4, p. 89 - 96, 2017. ISSN 2396-7544. <http://www.efortopenreviews.org/lookup/doi/10.1302/2058-5241.2. 160009>.

4 - MYGIND-KLAVSEN, B.; NIELSEN, T. G.; LIND, M. C. Outcomes after posterior cruciate ligament (PCL) reconstruction in patients with isolated and combined PCL tears. Orthopaedic Journal of Sports Medicine, v. 5, n. 4, p. 10 - 12, 2017. ISSN 23259671. 


\title{
RELATO DE EXPERIÊNCIA: CONSCIENTIZANDO A POPULAÇÃO SOBRE FIBROSE CÍSTICA
}

\author{
Camila Costa Ribeiro ${ }^{1}$; Pedro Kascher Silva ${ }^{1}$; Teresa Cristina Ribeiro Lopes ${ }^{1}$; Luciana \\ Carvalho dos Santos ${ }^{1}$; Mario Flavio Cardoso de Lima ${ }^{1}$; Marta Cristina Duarte ${ }^{2}$. \\ ${ }^{1}$ Acadêmicos de Medicina da Universidade Federal de Juiz de Fora (UFJF. \\ ${ }^{2}$ Docente da Faculdade de Medicina da Universidade Federal de Juiz de Fora.
}

Autor para correspondência:

Camila Costa Ribeiro

E-mail: ribeiro.camilla@medicina.ufjf.br

\section{RESUMO}

Introdução: Fibrose cística (FC) é uma doença genética que afeta uma a cada 10 mil pessoas no Brasil, sendo, portanto, uma das doenças raras mais comuns ${ }^{1}$. Apesar de estar incluída nas patologias rastreadas pelo teste do pezinho, a FC ainda é pouco conhecida pela população e é comumente confundida com outras afecções respiratórias como asma e pneumonia. Objetivo: Conscientizar a população de Juiz de Fora (JF) sobre FC.Relato de experiência: Foi realizada campanha de divulgação e conscientização da FC na primeira semana do mês de setembro de 2018, em locais de circulação pública na cidade de JF. A ação contou com a participação de 16 estudantes de medicina, orientados pela coordenadora local do Centro de Referência em FC. Os alunos, com o auxílio de materiais gráficos, orientaram transeuntes acerca dos sinais, sintomas, diagnóstico, tratamento e expectativas futuras com relação à doença. A experiência foi avaliada por meio de pesquisa qualitativa com os alunos participantes, que responderam às perguntas: "qual a parte mais interessante dessa experiência?" e "o que essa experiência acrescentou para você como profissional?". Resutados e discussão: O desconhecimento da doença pela população foi o tema mais destacado pelos alunos. "Foi bem interessante saber que mesmo a FC sendo rastreada pelo teste do pezinho, quase ninguém conhece. As pessoas fizeram muita confusão com doenças com nomes ou sintomas parecidos, todo mundo achava que algum parente tinha FC.". Os alunos também observaram que houve um interesse geral em aprender sobre a doença, especialmente ao saber sobre o Centro de Referência. "As pessoas se mostraram muito interessadas em ouvir e aprender sobre a FC, ficaram impressionadas ao saber que JF é referência no tratamento. Foi incrível ganhar a atenção das pessoas, que nos agradeceram e se comprometeram a divulgar a FC para conhecidos". Já na visão de futuro profissional de saúde, todos avaliaram positivamente o diálogo com a população. "Como profissional, tive a oportunidade não só de aprender mais sobre uma doença rara como também de traduzir o saber médico para o saber popular."; "É maravilhoso levar um pouco do conhecimento acadêmico para a população, especialmente em um tema importante como esse! Me sinto mais preparada para lidar com pacientes e futuros pacientes após essa semana de contato direto nas ruas.”. Conclusão: A conscientização sobre FC realizada em JF em 2018 demonstrou a importância de ações de estudantes e profissionais de saúde em conjunto com a população.

Palavras-chave: Fibrose Cística, sensibilização pública.

\section{Referências:}

1. FIRMIDA, M.C. LOPES, A.J. Aspectos Epidemiológicos da Fibrose Cística. Revista Hospital Universitário Pedro Ernesto, v 10, n 4, out/nov 2011. 


\title{
RELATO DE EXPERIÊNCIA: APRENDIZADO PRÁTICO DE DISCENTES DE MEDICINA EM CENTRO DE REFERÊNCIA EM FIBROSE CÍSTICA
}

\author{
Pedro Kascher Silva ${ }^{1}$; Camilla Costa Ribeiro ${ }^{1}$; Teresa Cristina Ribeiro Lopes ${ }^{1}$; Luciana \\ Carvalho dos Santos ${ }^{1}$; Mario Flavio Cardoso de Lima ${ }^{1}$; Marta Cristina Duarte ${ }^{2}$. \\ ${ }^{1}$ Acadêmicos de Medicina da Universidade Federal de Juiz de Fora (UFJF. \\ ${ }^{2}$ Docente da Faculdade de Medicina da Universidade Federal de Juiz de Fora.
}

\author{
Autor para correspondência: \\ Pedro Kascher Silva \\ E-mail: kascher.pedro@medicina.ufjf.br
}

\begin{abstract}
RESUMO
Introdução: Fibrose cística (FC) é uma doença genética multissistêmica, ainda sem cura. Pacientes com FC precisam ser acompanhados por equipe multidisciplinar apta a lidar com as manifestações dessa doença. Nesse sentido, a preparação profissional para a abordagem da FC deve ser estabelecida de forma precoce e integrada. Objetivo: Apresentar os resultados do treinamento profissional de acadêmicos de Medicina de uma universidade pública em Centro de Referência em Fibrose Cística (CRFC). Relato de experiência: O treinamento no CRFC foi realizado por dois estudantes de medicina (A1 e A2) durante dois anos, do terceiro ao sétimo período da graduação, e constituiu-se no acompanhamento de profissionais de saúde das áreas de medicina, psicologia, nutrição, fisioterapia, enfermagem, além da realização de consultas monitoradas por professor e da leitura periódica de artigos e consensos sobre FC. Após os dois anos, os alunos e a professora coordenadora do Centro $(\mathrm{C} 1)$ realizaram análise qualitativa dos conhecimentos e habilidades adquiridas a partir de eixos temáticos previamente estabelecidos, sendo eles: aprendizado sobre a doença; relação médico-paciente; e trabalho em equipe multidisciplinar. Resutados e discussão: Houve resposta positiva nos três eixos temáticos. Segundo A1: "É importante ressaltar o que aprendemos sobre a doença por si só, uma vez que é pouco falada no curso. O fato de você conviver com crianças com uma doença crônica rara, é uma experiência edificante, tanto como profissional de saúde quanto como ser humano." Segundo A2: "Além do que já foi falado, nosso contato multiprofissional foi muito importante para o desenvolvimento de espírito de trabalho em equipe e, principalmente, de valorização e respeito dos profissionais das diversas áreas que abrangem o tratamento." Segundo C1: "Em um primeiro momento, é digno de se ressaltar o interesse precoce de A1 e A2 pelo tema, considerando que este é complexo e pouco discutido na graduação. Além disso, foi possível perceber um aumento na produção científica do CRFC estimulada pelos próprios acadêmicos. Por fim, vejo a participação dos estudantes enquanto membros ativos no CRFC em consonância com a estratégia de Integração Ensino-Serviço que é, atualmente, um dos principais objetivos do planejamento da educação médica." Conclusão: A imersão precoce de acadêmicos de medicina em um CRFC é uma experiência positiva tanto para discentes quanto para docentes e outros profissionais, devendo esta prática ser estimulada.
\end{abstract}

Palavras-chave: Fibrose Cística; Educação Médica. 


\section{Referências:}

1. FIRMIDA, M.C. LOPES, A.J. Aspectos Epidemiológicos da Fibrose Cística. RevistaHospital Universitário Pedro Ernesto, v 10, n 4, out/nov 2011.

2. CASTEllani, C. ASSAEL, B.M. Cystic fibrosis: a clinical view. Cellular and Molecular Life Sciences, v 74, Issue 1, pp 129-140, January 2017.

3. DUARTE M.C. VERGARA, A.A. Fibrose Cística. In: Tratado das Enfermidades Gastrintestinais e Pancreáticas. Moraes-Filho JPP. São Paulo: Ed. Roca p.1204-1211,2008. 


\section{MELHORES TRABALHOS}

Os dois trabalhos melhores avaliados foram premiados pelo XIII Congresso Médico Acadêmicos. São eles:

- EDUCAÇÃo CONTRA O TABACO(EAT) NA PREVENÇÃO DO TABAGISMO: UM RELATO DE EXPERIÊNCIA (página 42);

- EMERGÊNCIAS MÉDICAS EM ODONTOLOGIA: A REALIDADE DAS GRADUAÇÕES EM MINAS GERAIS (página 16). 